\title{
STEIN STRUCTURES AND HOLOMORPHIC MAPPINGS
}

\author{
FRANC FORSTNERIČ \& MARKO SLAPAR
}

\begin{abstract}
We prove that every continuous map from a Stein manifold $X$ to a complex manifold $Y$ can be made holomorphic by a homotopic deformation of both the map and the Stein structure on $X$. In the absence of topological obstructions the holomorphic map may be chosen to have pointwise maximal rank. The analogous result holds for any compact Hausdorff family of maps, but it fails in general for a noncompact family. Our main results are actually proved for smooth almost complex source manifolds $(X, J)$ with the correct handlebody structure. The paper contains another proof of Eliashberg's (Int J Math 1:29-46, 1990) homotopy characterization of Stein manifolds and a slightly different explanation of the construction of exotic Stein surfaces due to Gompf (Ann Math 148 (2):619-693, 1998; J Symplectic Geom 3:565-587, 2005).
\end{abstract}

\section{INTRODUCTION}

A Stein manifold is a complex manifold which is biholomorphic to a closed complex submanifold of a Euclidean space $\mathbb{C}^{N}[40$. The following is a simplified version of our main results, Theorems 6.1, 6.2 and 7.1.

Theorem 1.1. Let $X$ be a Stein manifold with the complex structure operator $J$, and let $f: X \rightarrow Y$ be a continuous map to a complex manifold $Y$.

(i) If $\operatorname{dim}_{\mathbb{C}} X \neq 2$, there exist a Stein complex structure $\widetilde{J}$ on $X$, homotopic to $J$, and a $\widetilde{J}$-holomorphic map $\widetilde{f}: X \rightarrow Y$ homotopic to $f$.

(ii) If $\operatorname{dim}_{\mathbb{C}} X=2$, there are an orientation preserving homeomorphism $h: X \rightarrow X^{\prime}$ onto a Stein surface $X^{\prime}$ and a holomorphic map $f^{\prime}: X^{\prime} \rightarrow$ $Y$ such that the map $\tilde{f}:=f^{\prime} \circ h: X \rightarrow Y$ is homotopic to $f$.

The Stein structure $\widetilde{J}$ in (i), and the homeomorphism $h$ in (ii), can be chosen the same for all members of a compact Hausdorff family of maps $X \rightarrow Y$.

More precisely, in case (i) we find a smooth homotopy $J_{t} \in \operatorname{End}_{\mathbb{R}} T X$ $\left(J_{t}^{2}=-I d, t \in[0,1]\right)$ consisting of integrable (but not necessarily Stein) complex structures on the underlying smooth manifold $X$, connecting the

Date: July 5, 2006.

2000 Mathematics Subject Classification. 32H02, 32Q30, 32Q55, 32Q60, 32T15, 57R17.

Key words and phrases. Stein manifolds, complex structures, holomorphic mappings.

Supported by grants P1-0291 and J1-6173, Republic of Slovenia.

The original publication is available at http://www.springerlink.com.

http://dx.doi.org/10.1007/s00209-006-0093-0. 
Stein structure $J_{0}=J$ with a new Stein structure $J_{1}=\widetilde{J}$, such that $f$ is homotopic to a $\widetilde{J}$-holomorphic map. In case (ii) we get essentially the same statement after changing the smooth structure on $X$, i.e., the new Stein structure $\widetilde{J}$ on $X$ may be exotic. More precise statements are given by Theorem 6.1 for part (i), and by Theorem 7.1 for part (ii).

The question whether every continuous map from a Stein manifold to a given complex manifold $Y$ is homotopic to a holomorphic map is the central theme of the Oka-Grauert theory. Classical results of Oka [49], Grauert [30, 31, 32] and Gromov [37] give an affirmative answer when $Y$ is a complex homogeneous manifold or, more generally, if it admits a dominating spray. (See also [23] and [21].) Recently this Oka property of $Y$ has been characterized in terms of a Runge approximation property for entire maps $\mathbb{C}^{n} \rightarrow Y$ on certain special compact convex subset of $\mathbb{C}^{n}$ [19, 20]. The Oka property holds only rarely as it implies in particular that $Y$ is dominated by a complex Euclidean space, and this fails for any compact complex manifold of Kodaira general type. For a discussion of this subject see [21]. Although one cannot always find a holomorphic representative in each homotopy class of maps $X \rightarrow Y$, Theorem 1.1 gives a representative which is holomorphic with respect to some Stein structure on $X$ homotopic to the original one.

Even if the source complex manifold $X$ is not Stein, we can obtain a holomorphic map in a given homotopy class on a suitable Stein domain in $X$, provided that $X$ has a correct handlebody structure.

Theorem 1.2. Let $X$ be an $n$-dimensional complex manifold which admits a Morse exhaustion function $\rho: X \rightarrow \mathbb{R}$ without critical points of index $>n$. Let $f: X \rightarrow Y$ be continuous map to a complex manifold $Y$.

(i) If $n \neq 2$, there exist an open Stein domain $\Omega$ in $X$, a diffeomorphism $h: X \rightarrow h(X)=\Omega$ which is diffeotopic to the identity map $i d_{X}$ on $X$, and a holomorphic map $f^{\prime}: \Omega \rightarrow Y$ such that $f^{\prime} \circ h: X \rightarrow Y$ is homotopic to $f$.

(ii) If $n=2$, the conclusion in (i) still holds if $\rho$ has no critical points of index $>1$; in the presence of critical points of index 2 the conclusion holds with $h$ a homeomorphism which is homeotopic to $i d_{X}$.

Theorem 1.2 immediately implies Theorem 1.1: If $h_{t}: X \rightarrow h_{t}(X) \subset X$ $(t \in[0,1])$ is a diffeotopy from $h_{0}=i d_{X}$ to $h_{1}=h: X \rightarrow \Omega$ as in Theorem 1.2 then $J_{t}:=h_{t}^{*}(J)$ is a homotopy of integrable complex structures on $X$ connecting the original structure $J_{0}=J$ to a Stein structure $\widetilde{J}=h^{*}\left(\left.J\right|_{T \Omega}\right)$, and $\widetilde{f}=f^{\prime} \circ h: X \rightarrow Y$ is a $\widetilde{J}$-holomorphic map homotopic to $f$.

Our proof of Theorem 1.2 shows that the only essential obstruction in finding a holomorphic map in a given homotopy class is that $X$ may be holomorphically 'too large' to fit into $Y$. This vague notion of 'holomorphic rigidity' has several concrete manifestations, for example, the distance decreasing property of holomorphic maps in most of the standard biholomorphically invariant metrics (such as Kobayashi's). The problem can be 
avoided by restricting the size of the domain while at the same time retaining the topological (and smooth in dimension $\neq 2$ ) characteristics of $X$.

The following simple example illustrates that Theorems 1.1 and 1.2 are optimal even for maps of Riemann surfaces.

Example 1.3. Let $X=A_{r}=\{z \in \mathbb{C}: 1 / r<|z|<r\}$, and let $Y=A_{R}$ for another $R>1$. We have $[X, Y]=\mathbb{Z}$. A homotopy class represented by an integer $k \in \mathbb{Z}$ admits a holomorphic representative if and only if $r^{|k|} \leq R$, and in this case a representative is $z \rightarrow z^{k}$. Since every complex structure on an annulus is biholomorphic to $A_{r}$ for some $r>1$, we see that at most finitely many homotopy classes of maps between any pair of annuli contain a holomorphic map. The conclusion of Theorem 1.1 can be obtained by a radial dilation, decreasing the value of $r>1$ to another value satisfying $r^{k} \leq R$, which amounts to a homotopic change of the complex structure on $X$. This allows us to simultaneously deform any compact family of maps $X \rightarrow Y$ to a family of holomorphic maps, but it is impossible to do it for a sequence of maps belonging to infinitely many different homotopy classes. The problem disappears in the limit as $R=+\infty$ when $Y$ is the complex Lie group $\mathbb{C}^{*}=\mathbb{C} \backslash\{0\}$ and the Oka-Grauert principle applies [31, [49]. The same phenomenon appears whenever the fundamental group $\pi_{1}(Y)$ contains an element $[\alpha]$ of infinite order such that the minimal Kobayashi length $l_{N}$ of loops in $Y$ representing the class $N[\alpha] \in \pi_{1}(Y)$ tends to $+\infty$ as $N \rightarrow+\infty$ : A homotopically nontrivial loop $\gamma$ in $X$ with positive Kobayashi length $K_{X}(\gamma)$ can be mapped to the class $N[\alpha]$ by a holomorphic map $X \rightarrow Y$ only if $l_{N} \leq K_{X}(\gamma)$, and this is possible for at most finitely many $N \in \mathbb{N}$.

Our construction also gives holomorphic maps of maximal rank (immersions resp. submersions) provided that there are no topological obstructions. The following is a simplified version of Theorem 6.2 below.

Theorem 1.4. Let $X$ be a Stein manifold of dimension $\operatorname{dim} X \neq 2$. Assume that $f: X \rightarrow Y$ is a continuous map to a complex manifold $Y$ which is covered by a complex vector bundle map $\iota: T X \rightarrow f^{*}(T Y)$ of fiberwise maximal rank. Then there is a Stein structure $\widetilde{J}$ on $X$, homotopic to $J$, and a $\widetilde{J}$-holomorphic map $\tilde{f}: X \rightarrow Y$ of pointwise maximal rank which is homotopic to $f$. The analogous conclusion holds if $\operatorname{dim} X=2$ and $X$ admits a Morse exhaustion function $\rho: X \rightarrow \mathbb{R}$ without critical points of index $>1$.

Theorem 1.4 is a holomorphic analogue of the Smale-Hirsch h-principle for smooth immersions [54, 39, 36] and of the Gromov-Phillips h-principle for smooth submersions [34, 51]. The conclusion holds with a fixed Stein structure on $X$ provided that $Y$ satisfies a certain flexibility condition introduced (for submersions) in [18]. For maps to Euclidean spaces see also [36, $\S 2.1 .5]$ (for immersions) and [17] (for submersions).

An important source of Stein manifolds are the holomorphically complete Riemann domains $\pi: X \rightarrow \mathbb{C}^{n}, \pi$ being a locally biholomorphic map. These 
arise as the envelopes of holomorphy of domains in, or over, $\mathbb{C}^{n}$. Clearly every such manifold is holomorphically parallelizable, but the converse has been a long standing open problem:

Does every $n$-dimensional Stein manifold $X$ with a trivial complex tangent bundle admit a locally biholomorphic map $\pi: X \rightarrow \mathbb{C}^{n}$ ?

In 1967 Gunning and Narasimhan gave a positive answer for open Riemann surfaces [38]. In 2003 the first author of this paper proved that every parallelizable Stein manifold $X^{n}$ admits a holomorphic submersion $f: X \rightarrow \mathbb{C}^{n-1}$ [17]; the remaining problem is to find a holomorphic function $g$ on $X$ whose restriction to each level set of $f$ has no critical points (the map $(f, g): X \rightarrow \mathbb{C}^{n}$ is then locally biholomorphic). Theorem 1.4 with $Y=\mathbb{C}^{n}$ $(n=\operatorname{dim} X)$ shows that the above problem is solvable up to homotopy:

Corollary 1.5. If $(X, J)$ is a Stein manifold of dimension $n \neq 2$ whose holomorphic tangent bundle $T X$ is trivial then there are a Stein structure $\widetilde{J}$ on $X$, homotopic to $J$, and a $\widetilde{J}$-holomorphic immersion $\pi: X \rightarrow \mathbb{C}^{n}$.

Note that every closed complex submanifold $X \subset \mathbb{C}^{N}$ with trivial complex normal bundle $\left.T \mathbb{C}^{N}\right|_{X} / T X$ is parallelizable [13]; this holds in particular for any smooth complex hypersurface in $\mathbb{C}^{N}$.

All our main results are actually proved in the class of smooth almost complex manifolds $(X, J)$ which admit a Morse exhaustion function $\rho: X \rightarrow$ $\mathbb{R}$ without critical points of index $>n=\frac{1}{2} \operatorname{dim}_{\mathbb{R}} X$ (see Theorems 6.1, 6.2 and 7.1). By Morse theory such $X$ is homotopically equivalent to a CW complex of dimension at most $n$ [46. Since the Morse indices of any strongly plurisubharmonic exhaustion function satisfy this index condition, this holds for every Stein manifold (Lefshetz [44, Andreotti and Fraenkel [1], Milnor [46]). Conversely, if $(X, J)$ satisfies the above index condition and $\operatorname{dim}_{\mathbb{R}} X \neq 4$ then $J$ is homotopic to an integrable Stein structure on $X$ according to Eliashberg [7]. The present paper contains another proof of this important result, with an additional argument provided in the critical case when attaching handles of maximal real dimension $n=\frac{1}{2} \operatorname{dim}_{\mathbb{R}} X$. A detailed understanding of this construction is unavoidable for our purposes, and assuming that the initial structure on $X$ is already integrable Stein (as in Theorem 1.1) does not really simplify our proof.

The story is even more interesting when $\operatorname{dim}_{\mathbb{R}} X=4: A$ smooth oriented four manifold without handles of index $>2$ is homeomorphic to a Stein surface, but the underlying smooth structure must be changed in general (Gompf [27, 28]). Indeed, a closed orientable real surface $S$ smoothly embedded in a Stein surface $X$ (or in a compact Kähler surface with $b^{+}(X)>1$ ), with the only exception of a null-homologous 2-sphere, satisfies the generalized adjunction inequality:

$$
[S]^{2}+\left|c_{1}(X) \cdot S\right| \leq-\chi(S)
$$


(See Chapter 11 in 29 and the papers [16, 42, 45, 47, 50.) For a 2-sphere the above inequality yields $[S]^{2} \leq-2$. Taking $X=S^{2} \times \mathbb{R}^{2}=\mathbb{C P}^{1} \times \mathbb{C}$, the embedded 2-sphere $S^{2} \times\{0\} \subset X$ generates $H_{2}(X, \mathbb{Z})=\mathbb{Z}$ and satisfies $[S]^{2}=0$, hence $X$ does not admit any non-exotic Stein structure.

Nevertheless, there is a bounded Stein domain in $\mathbb{C}^{2}$ homeomorphic to $S^{2} \times \mathbb{R}^{2}$. This is a special case of Gompf's result that for every tamely topologically embedded CW 2-complex $M$ in a complex surface $X$ there exists a topological isotopy of $X$ which is uniformly close to the identity on $X$ and which carries $M$ onto a complex $M^{\prime} \subset X$ with a Stein thickening, i.e., an open Stein domain $\Omega \subset X$ homeomorphic to the interior of a handlebody with core $M$ [28, Theorem 2.4]. In his proof, Gompf uses kinky handles of index 2 in each place where an embedded 2-handle with suitable properties cannot be found in Eliashberg's construction. To obtain the correct manifold one must perform an inductive procedure which cancels all superfluous loops caused by kinks, thereby creating Casson handles which are homeomorphic, but not diffeomorphic, to the standard index two handle $D^{2} \times D^{2}$ (Freedman [25]). In $\S 7$ we follow a similar path to construct a holomorphic map in the chosen homotopy class, performing the Casson tower construction simultaneously at a possibly increasing number of places.

Organization of the paper. In $\S 2$ we recall the relevant notions from Stein and contact geometry. Sections $\S 3-\S 5$ contain preparatory lemmas. The main geometric ingredient is Lemma 3.1 which gives totally real discs attached from the exterior to a strongly pseudoconvex domain along a complex tangential sphere. A main analytic ingredient is an approximation theorem for holomorphic maps to arbitrary complex manifolds (Theorem 4.1). Lemma 5.1 provides an approximate extension of a holomorphic map to an attached handle. The main results are presented and proved in sections $\S 6$ (for $\operatorname{dim}_{\mathbb{C}} X \neq 2$ ) and $\S 7$ (for $\operatorname{dim}_{\mathbb{C}} X=2$ ).

\section{Preliminaries}

We begin by recalling some basic notions of the handlebody theory; see e.g. [26], 29], 46]. Let $X$ be a smooth compact $n$-manifold with boundary $\partial X$, and let $D^{k}$ denote the closed unit ball in $\mathbb{R}^{k}$. A $k$-handle $H$ attached to $X$ is a copy of $D^{k} \times D^{n-k}$ smoothly attached to $\partial X$ along $\partial D^{k} \times D^{n-k}$, with the corners smoothed, which gives a larger compact manifold with boundary. The central disc $D^{k} \times\{0\}^{n-k}$ is the core of $H$. A handle decomposition of a smooth (open or closed) manifold $X$ is a representation of $X$ as an increasing union of compact domains with boundary $X_{j} \subset X$ such that $X_{j+1}$ is obtained by attaching a handle to $X_{j}$. (In the case of open manifolds one takes the interior of the resulting handlebody.) By Morse theory every smooth manifold admits a handlebody representation.

An almost complex structure on an even dimensional smooth manifold $X$ is a smooth endomorphism $J \in \operatorname{End}_{\mathbb{R}}(T M)$ satisfying $J^{2}=-I d$. The 
operator $J$ gives rise to the conjugate differential $d^{c}$, defined on functions by $\left\langle d^{c} \rho, v\right\rangle=-\langle d \rho, J v\rangle$ for $v \in T X$, and the Levi form operator $d d^{c} . J$ is said to be integrable if every point of $X$ admits an open neighborhood $U \subset X$ and a $J$-holomorphic coordinate map of maximal rank $z=\left(z_{1}, \ldots, z_{n}\right): U \rightarrow \mathbb{C}^{n}$ $\left(n=\frac{1}{2} \operatorname{dim}_{\mathbb{R}} X\right)$, i.e., satisfying $d z \circ J=i d z$; for a necessary and sufficient integrability condition see Newlander and Nirenberg [48].

If $h: X \rightarrow X^{\prime}$ is a diffeomorphism and $J^{\prime}$ is an almost complex structure on $X^{\prime}$, we denote by $J=h^{*}\left(J^{\prime}\right)$ the (unique) almost complex structure on $X$ satisfying $d h \circ J=J^{\prime} \circ d h$; i.e., such that $h$ is a biholomorphism. Similarly we denote by $J^{\prime}=h_{*}(J)$ the push-forward of an almost complex structure $J$ by $h$. A map $f^{\prime}: X^{\prime} \rightarrow Y$ to a complex manifold $Y$ is $J^{\prime}$-holomorphic if and only if $f=f^{\prime} \circ h: X \rightarrow Y$ is $J$-holomorphic with $J=h^{*}\left(J^{\prime}\right)$.

An integrable structure $J$ on a smooth manifold $X$ is said to be Stein if $(X, J)$ is a Stein manifold; this is the case if and only if there is a strongly $J$ plurisubharmonic Morse exhaustion function $\rho: X \rightarrow \mathbb{R}$, i.e., $\left\langle d d^{c} \rho, v \wedge J v\right\rangle>$ 0 for every $0 \neq v \in T X$ (Grauert [33]). The $(1,1)$-form $\omega=d d^{c} \rho=2 i \partial \bar{\partial} \rho$ is then a symplectic form on $X$, defining a $J$-invariant Riemannian metric $g(v, w)=\langle\omega, v \wedge J w\rangle(v, w \in T X)$. The Morse indices of such function $\rho$ are $\leq n=\frac{1}{2} \operatorname{dim}_{\mathbb{R}} X$ and hence $X$ is the interior of a handlebody without handles of index $>n$ [1, 46].

A real subbundle $V$ of the tangent bundle $T X$ is said to be $J$-real, or totally real, if $V_{x} \cap J V_{x}=\{0\}$ for every $x \in X$; its complexification $V^{\mathbb{C}}=$ $V \otimes_{\mathbb{R}} \mathbb{C}$ can be identified with the $J$-complex subbundle $V \oplus J V$ of $T X$. An immersion $G: D \rightarrow X$ of a smooth manifold $D$ into $X$ is $J$-real (or totally real) if $d G_{x}\left(T_{x} D\right)$ is a $J$-real subspace of $T_{G(x)} X$ for every $x \in D$.

Let $W$ be a relatively compact domain with smooth boundary $\Sigma=\partial W$ in an almost complex manifold $(X, J)$. The set $\xi=T \Sigma \cap J(T \Sigma)$ is a corank one $J$-complex linear subbundle of $T \Sigma$. Assume now that $\rho$ is a smooth function in a neighborhood of $\Sigma=\partial W$ such that $\Sigma=\{\rho=0\}, d \rho \neq 0$ on $\Sigma$ and $\rho<0$ on $W$. Let $\eta:=\left.d^{c} \rho\right|_{T \Sigma}$, a one-form on $\Sigma$ with ker $\eta=\xi$. We say that $\Sigma$ is strongly $J$-pseudoconvex, or simply $J$-convex, if $\left\langle d d^{c} \rho, v \wedge J v\right\rangle>0$ for all $0 \neq v \in \xi$; this condition is independent of the choice of $\rho$. (We shall omit $J$ when it is clear which almost complex structure do we have in mind.) This implies that $\eta \wedge(d \eta)^{n-1} \neq 0$ on $\Sigma\left(n=\operatorname{dim}_{\mathbb{C}} X\right)$ which means that $\eta$ is a contact form and $(\Sigma, \xi)$ is a contact manifold (see [6, 7, 36, pp. 338-340]). A smooth function $\rho: X \rightarrow \mathbb{R}$ whose level sets are $J$-convex outside of the critical points is said to be $J$-convex.

An immersion $g: S \rightarrow \Sigma$ of a smooth manifold $S$ into a contact manifold $(\Sigma, \xi)$ is Legendrian if $d g(T S) \subset \xi$. In the case at hand, when $\Sigma$ is the boundary of a strongly pseudoconvex domain, another common expression is a complex tangential immersion.

Let $J_{s t}$ denote the standard complex structure on $\mathbb{C}^{n}$. For a fixed $k \in$ $\{1, \ldots, n\}$ let $z=\left(z_{1}, \ldots, z_{n}\right)=\left(x^{\prime}+i y^{\prime}, x^{\prime \prime}+i y^{\prime \prime}\right)$, with $z_{j}=x_{j}+i y_{j}$, 
denote the coordinates on $\mathbb{C}^{n}$ corresponding to the decomposition

$$
\mathbb{C}^{n}=\mathbb{C}^{k} \oplus \mathbb{C}^{n-k}=\mathbb{R}^{k} \oplus i \mathbb{R}^{k} \oplus \mathbb{R}^{n-k} \oplus i \mathbb{R}^{n-k} .
$$

Let $D=D^{k} \subset \mathbb{R}^{k}$ be the closed unit ball in $\mathbb{R}^{k}$ and $S=S^{k-1}=\partial D$ its boundary sphere. Identifying $D^{k}$ with its image in $\mathbb{R}^{k} \oplus\{0\}^{2 n-k} \subset \mathbb{C}^{n}$ we obtain the core of the standard index $k$ handle

$$
H_{\delta}=(1+\delta) D^{k} \times \delta D^{2 n-k} \subset \mathbb{C}^{n}, \quad \delta>0 .
$$

A standard handlebody of index $k$ in $\mathbb{C}^{n}$ is a set $K_{\lambda, \delta}=Q_{\lambda} \cup H_{\delta}$ for some $0<\lambda<1$ and $0<\delta<\frac{2 \lambda}{1-\lambda}$ (Fig. 1), where

$$
Q_{\lambda}=\left\{z=\left(x^{\prime}+i y^{\prime}, z^{\prime \prime}\right) \in \mathbb{C}^{k} \oplus \mathbb{C}^{n-k}:\left|y^{\prime}\right|^{2}+\left|z^{\prime \prime}\right|^{2} \leq \lambda\left(\left|x^{\prime}\right|^{2}-1\right)\right\}
$$

The condition $\lambda<1$ insures that $Q_{\lambda}$ is strongly pseudoconvex, and the bound on $\delta$ implies $(1+\delta) \partial D^{k} \times \delta D^{2 n-k} \subset Q_{\lambda}$.

We shall need the following result of Eliashberg [7, §3]. (See also [22].)

Lemma 2.1. (Eliashberg) For every $\epsilon>0$ and $\lambda>1$ there exist a number $\delta \in(0, \epsilon)$ and a smoothly bounded, strongly pseudoconvex handlebody $L \subset \mathbb{C}^{n}$ with core $Q_{\lambda} \cup D^{k}$ such that $K_{\lambda, \delta} \subset L \subset K_{\lambda, \epsilon}$ (Fig. 1).

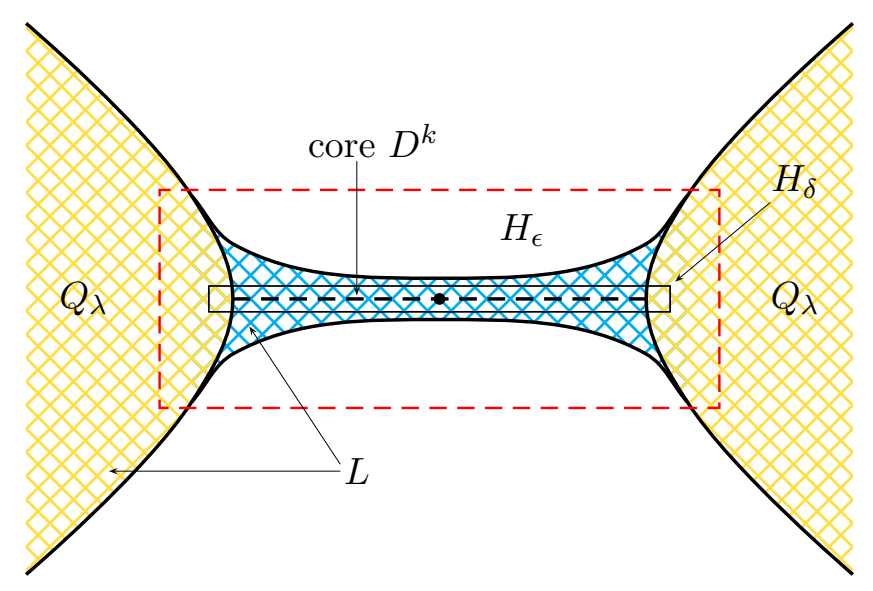

Figure 1. A strongly pseudoconvex handlebody $L$

Eliashberg's construction gives an $L$ of the form

$$
L=\left\{\left(x^{\prime}+i y^{\prime}, z^{\prime \prime}\right) \in \mathbb{C}^{n}:\left|y^{\prime}\right|^{2}+\left|z^{\prime \prime}\right|^{2} \leq h\left(\left|x^{\prime}\right|^{2}\right)\right\}
$$

where $h:[0, \infty] \rightarrow\left[\delta^{2}, \infty\right]$ is a smooth, increasing, convex function chosen so that $L$ is a tube of constant radius $\delta$ around $D^{k} \subset \mathbb{C}^{n}$ over a slightly smaller ball $r D^{k}(r<1)$, and $L$ equals $Q_{\lambda}$ over $r^{\prime} D^{k}$ for some $r^{\prime}>1$ close to 1 . 
We introduce the following (trivial) bundles over the disc $D \subset \mathbb{R}^{k} \oplus$ $\{0\}^{2 n-k}$ :

$$
\begin{aligned}
\nu^{\prime} & =\left.\operatorname{Span}\left\{\frac{\partial}{\partial y_{1}}, \ldots, \frac{\partial}{\partial y_{k}}\right\}\right|_{D}=D \times\left(\{0\}^{k} \oplus \mathbb{R}^{k} \oplus\{0\}^{2 n-2 k}\right), \\
\nu^{\prime \prime} & =\left.\operatorname{Span}\left\{\frac{\partial}{\partial x_{j}}, \frac{\partial}{\partial y_{j}}: j=k+1, \ldots, n\right\}\right|_{D}=D \times\left(\{0\}^{2 k} \oplus \mathbb{R}^{2 n-2 k}\right), \\
\nu & =\nu^{\prime} \oplus \nu^{\prime \prime}=D \times\left(\{0\}^{k} \oplus \mathbb{R}^{2 n-k}\right) .
\end{aligned}
$$

Thus $\nu^{\prime}=J_{s t}(T D), T^{\mathbb{C}} D=T D \oplus \nu^{\prime}$, and $\left.T \mathbb{C}^{n}\right|_{D}=T D \oplus \nu=T^{\mathbb{C}} D \oplus \nu^{\prime \prime}$.

Let $v \rightarrow S$ denote the (trivial) real line bundle over $S$ spanned by the vector field $\sum_{j=1}^{k} x_{j} \frac{\partial}{\partial x_{j}}$. Over $S$ we then have further decompositions

$$
\left.T D\right|_{S}=v \oplus T S,\left.\quad \nu^{\prime}\right|_{S}=J_{s t}(v) \oplus J_{s t}(T S),\left.\left.\quad T D\right|_{S} \oplus \nu^{\prime}\right|_{S} \simeq v^{\mathbb{C}} \oplus T^{\mathbb{C}} S .
$$

Note that $T^{\mathbb{C}} S$ is a trivial complex vector bundle.

Given a smooth embedding (or immersion) $G: D \rightarrow X$ of the disc $D=$ $D^{k} \subset \mathbb{C}^{n}$ to a smooth $2 n$-dimensional manifold $X$, a normal framing over $G$ is a homomorphism $\beta:\left.\nu \rightarrow T X\right|_{G(D)}$ such that $d G_{x} \oplus \beta_{x}: T_{x} D \oplus \nu_{x}=$ $T_{x} \mathbb{C}^{n} \rightarrow T_{G(x)} X$ is a linear isomorphism for every $x \in D$.

\section{Totally REAl Discs ATtaChed to STRONGLY PSEUdoconVEX DOMAINS ALONG LEGENDRIAN SPHERES}

Let $W$ be an open, relatively compact domain with smooth strongly pseudoconvex boundary $\Sigma=\partial W$ in an almost complex manifold $(X, J)$ of real dimension $2 n$. Choose a smooth defining function $\rho$ for $W$ which is strongly $J$-plurisubharmonic near $\Sigma=\{\rho=0\}$. Let $\left.w \subset T X\right|_{\Sigma}$ be the orthogonal complement of $T \Sigma$ with respect to the metric associated to the symplectic form $d d^{c} \rho$ (see $\S 2$ ); thus $w$ is spanned by the gradient of $\rho$ with respect to this metric. Then $J w \subset T \Sigma$ and we have orthogonal decompositions $\left.T X\right|_{\Sigma}=w \oplus T \Sigma=w \oplus J w \oplus \xi$, where $\xi=T \Sigma \cap J(T \Sigma)$.

Let $D=D^{k}, S=S^{k-1}=\partial D$ and $v$ be as in $\S 2$. An embedding of a pair $G:(D, S) \rightarrow(X \backslash W, \Sigma)$ is a smooth embedding $G: D \hookrightarrow X \backslash W$ such that $G(S)=G(D) \cap \Sigma$ and $G$ is transverse to $\Sigma$ along $G(S)$. Such $G$ is said to be normal to $\Sigma$ if $d G_{x}\left(v_{x}\right)=w_{G(x)}$ for every $x \in S$, i.e., $G$ maps the direction orthogonal to $S \subset \mathbb{R}^{k}$ into the direction orthogonal to $\Sigma \subset X$. The analogous definition applies to immersions.

The following lemma is a key geometric ingredient in the proof of all main results in this paper. Its proof closely follows the construction of a special handle attaching triple (HAT) in $§ 2$ of Eliashberg's paper [7, but with an additional argument in the critical case $k=n \neq 2$ (see Remark 3.2 below). We thank Y. Eliashberg for his help in the proof of the critical case (private communication, June 2005). 
Lemma 3.1. Let $W$ be an open, relatively compact domain with smooth strongly pseudoconvex boundary $\Sigma=\partial W$ in an almost complex manifold $(X, J)$. Let $1 \leq k \leq n=\frac{1}{2} \operatorname{dim}_{\mathbb{R}} X, D=D^{k}, S=\partial D$. Given a smooth embedding $G_{0}:(D, S) \rightarrow(X \backslash W, \Sigma)$, there is a regular homotopy of immersions $G_{t}:(D, S) \rightarrow(X \backslash W, \Sigma)(t \in[0,1])$ which is $\mathcal{C}^{0}$ close to $G_{0}$ such that the immersion $G_{1}: D \rightarrow X \backslash W$ is $J$-real and normal to $\Sigma$, and $g_{1}:=\left.G_{1}\right|_{S}: S \hookrightarrow \Sigma$ is a Legendrian embedding. If $k<n$, or if $k=n \neq 2$, there exists an isotopy of embeddings $G_{t}$ with these properties. If $J$ is integrable in a neighborhood of $\Sigma \cup G_{0}(D)$ and $\Sigma$ is real-analytic then $G_{1}$ can be chosen real analytic.

As was pointed out in [7, Note 2.4.2.], the topological obstruction in the case $k=n=2$ is essential. For example, there does not exist an embedded totally real 2-disc in $\mathbb{C}^{2} \backslash B$, attached to the ball $B \subset \mathbb{C}^{2}$ along a Legendrian curve in $\partial B$, since by [7] the resulting configuration would admit an open Stein neighborhood diffeomorphic to $S^{2} \times \mathbb{R}^{2}$ in contradiction to the generalized adjunction inequality (1.1).

Proof. The scheme of proof is illustrated on Fig. 2, First we find a regular homotopy from the initial disc $G_{0}: D \hookrightarrow X \backslash W$ to an immersed disc $G_{1}: D \rightarrow X \backslash W$ which is attached with a correct normal framing to $\partial W$ along an embedded Legendrian sphere. Next we deform $G_{1}$ by a regular homotopy which is fixed near the boundary to a totally real immersed disc $G_{2}$, using the h-principle for totally real immersions. Finally we show that, unless $k=n=2$, the construction can be done by isotopies of embeddings.

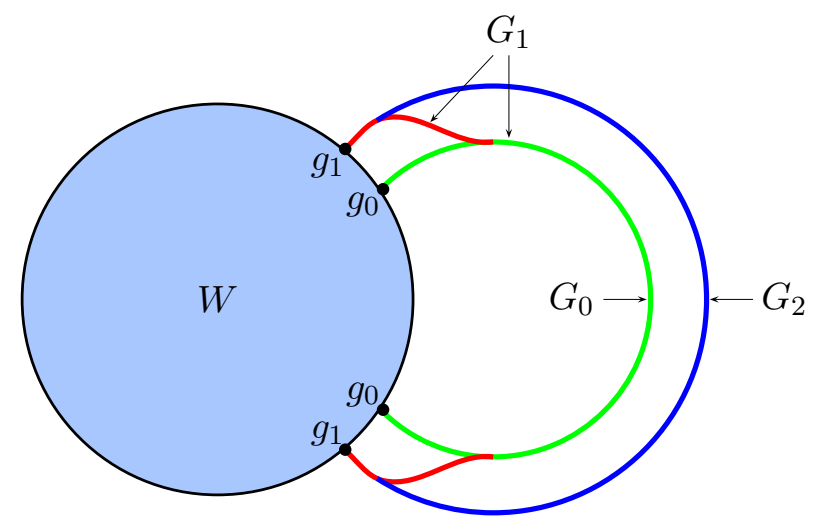

Figure 2. Deformations of an attached disc

Set $g_{0}=\left.G_{0}\right|_{S}: S \hookrightarrow \partial W$. By a correction of $G_{0}$ along $S$ (keeping $g_{0}$ fixed) we may assume that it is normal to $\Sigma$, i.e., such that $l_{0}:=\left.d G_{0}\right|_{v}$ maps $v$ to $\left.w\right|_{g_{0}(S)}$. Choose a complex vector bundle isomorphism

$$
\phi_{0}:\left.T \mathbb{C}^{n}\right|_{D}=D \times\left.\mathbb{C}^{n} \rightarrow T X\right|_{G_{0}(D)}, \quad \phi_{0} \circ J_{s t}=J \circ \phi_{0}
$$


covering $G_{0}$. We shall use the coordinates on $\mathbb{C}^{n}$ introduced in $\S 2$. The vector field $\tau=\sum_{j=1}^{k} x_{j} \frac{\partial}{\partial x_{j}}$ is outer radial to the sphere $S=\partial D$ in $\mathbb{R}^{k} \times\{0\}^{2 n-k}$. Let $\widetilde{\tau}$ be the unique nonvanishing vector field on $\mathbb{C}^{n}$ over $S$ satisfying $\phi_{0}\left(\widetilde{\tau}_{x}\right)=\ell_{0}\left(\tau_{x}\right)$ for every $x \in S$. By dimension reasons there exists a map $A: D \rightarrow G L_{n}(\mathbb{C})$ satisfying $A_{x} \tau_{x}=\widetilde{\tau}_{x}$ for $x \in S$. Replacing $\phi_{0}$ by $\phi_{0} \circ A$ we may (and shall) assume from now on that $\left.\phi_{0}\right|_{v}=\ell_{0}$. A further homotopic correction of $\phi_{0}$ insures that $\phi_{0}\left(\left.T^{\mathbb{C}} S \oplus \nu^{\prime \prime}\right|_{S}\right)=\left.\xi\right|_{g_{0}(S)}$, thereby providing a trivialization of the latter bundle.

Write $\phi_{0}=\phi_{0}^{\prime} \oplus \phi_{0}^{\prime \prime}$ where $\phi_{0}^{\prime}=\left.\phi_{0}\right|_{T^{\mathbb{C}} D}$ and $\phi_{0}^{\prime \prime}=\left.\phi_{0}\right|_{\nu^{\prime \prime}}$ (we use the notation of $\S 2$ ). Setting $\psi_{0}:=\left.\phi_{0}\right|_{T^{\mathbb{C}} S}$ we thus have

$$
\left.\phi_{0}^{\prime}\right|_{\left.T^{\mathbb{C}} D\right|_{S}}=\ell_{0}^{\mathbb{C}} \oplus \psi_{0}:\left.v^{\mathbb{C}} \oplus T^{\mathbb{C}} S \rightarrow T X\right|_{g_{0}(S)}=\left.w^{\mathbb{C}} \oplus \xi\right|_{g_{0}(S)} .
$$

Note that $\psi_{0} \oplus \phi_{0}^{\prime \prime}:\left.\left.T^{\mathbb{C}} S \oplus \nu^{\prime \prime}\right|_{S} \rightarrow \xi\right|_{g_{0}(S)}$ is a complex vector bundle isomorphism. Furthermore, there is a homotopy of real vector bundle monomorphisms $\iota_{s}:\left.T D \hookrightarrow T X\right|_{G_{0}(D)}(s \in[0,1])$ satisfying

$$
\iota_{0}=d G_{0}, \quad \iota_{1}=\left.\phi_{0}\right|_{T D},\left.\quad \iota_{s}\right|_{v}=\ell_{0}:\left.v \rightarrow w\right|_{g_{0}(S)} \quad(s \in[0,1]) .
$$

Consider the pair $\left(g_{0}, \psi_{0}\right)$ consisting of the embedding $g_{0}: S \hookrightarrow \Sigma$ and the $\mathbb{C}$-linear embedding $\psi_{0}:\left.T^{\mathbb{C}} S \hookrightarrow \xi\right|_{g_{0}(S)}$ of the complexified tangent bundle of $S$ (a trivial complex vector bundle of rank $k-1$ ) into the contact subbundle $\xi \subset T \Sigma$ over the map $g_{0}$. By the Legendrization theorem of Gromov ([36], p. 339, (B')) and Duchamp [6] there exists a Legendrian embedding $g_{1}: S \hookrightarrow \Sigma$ whose complexified differential $\psi_{1}:=d^{\mathbb{C}} g_{1}$ is homotopic to $\psi_{0}$ by a homotopy of $\mathbb{C}$-linear vector bundle embeddings $\psi_{t}: T^{\mathbb{C}} S \hookrightarrow \xi(t \in[0,1])$.

Let $\operatorname{Hom}_{i n j}(T S, T \Sigma)$ denote the space of all fiberwise injective real vector bundle maps $T S \hookrightarrow T \Sigma$. Consider the path in $\operatorname{Hom}_{i n j}(T S, T \Sigma)$ beginning at $d g_{0}$ and ending at $d g_{1}$, consisting of the homotopy $\left.\iota_{s}\right|_{T S}(s \in[0,1])$ followed by the homotopy $\left.\psi_{t}\right|_{T S}(t \in[0,1])$ (left and top side of the square in Fig. 3). By Hirsch's one parametric h-principle for immersions [36, 39] this path can be deformed in the space $\operatorname{Hom}_{i n j}(T S, T \Sigma)$ (with fixed ends) to a path $d g_{t}:\left.T S \hookrightarrow T \Sigma\right|_{g_{t}(S)}$ where $g_{t}: S \rightarrow \Sigma(t \in[0,1])$ is a regular homotopy of immersions from $g_{0}$ to $g_{1}$. We can insure that $\psi_{t}$ covers the base map $g_{t}$ for all $t \in[0,1]$. This gives a two parameter homotopy $\theta_{t, s} \in \operatorname{Hom}_{i n j}(T S, T \Sigma)$ for $(t, s) \in[0,1]^{2}$ satisfying the following conditions (Fig. 33):

(i) $\theta_{t, 0}=d g_{t}$ (bottom side),

(ii) $\theta_{t, 1}=\left.\psi_{t}\right|_{T S}$ (top side),

(iii) $\theta_{0, s}=\left.\iota_{s}\right|_{T S}$ (left side; hence $\theta_{0,0}=d g_{0}$ and $\theta_{0,1}=\left.\psi_{0}\right|_{T S}$ ),

(iv) $\theta_{1, s}=d g_{1}$ (right side), and

(v) $\theta_{t, s}$ covers $g_{t}$ for every $t, s \in[0,1]$.

We can extend $g_{t}$ to a regular homotopy of immersions $G_{t}:(D, S) \rightarrow$ $(X \backslash W, \Sigma)(t \in[0,1])$ which are normal to $\Sigma$, beginning at $t=0$ with the given map $G_{0}$. Let $\ell_{t}:=\left.d G_{t}\right|_{v}:\left.v \rightarrow w\right|_{g_{t}(S)}$. By the homotopy lifting 


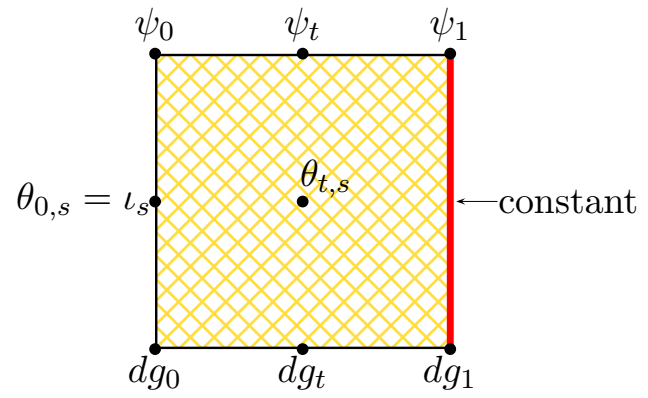

FIGURE 3. The homotopy $\theta_{t, s}$

theorem there exists a homotopy of $\mathbb{C}$-linear vector bundle isomorphisms $\phi_{t}$ covering $G_{t}$,

$$
\phi_{t}=\phi_{t}^{\prime} \oplus \phi_{t}^{\prime \prime}:\left.T \mathbb{C}^{n}\right|_{D}=\left.T^{\mathbb{C}} D \oplus \nu^{\prime \prime} \rightarrow T X\right|_{G_{t}(D)}, \quad t \in[0,1],
$$

beginning at $t=0$ with the given map $\phi_{0}$, such that over $S=\partial D$ we have

$$
\phi_{t}^{\prime}=\ell_{t}^{\mathbb{C}} \oplus \psi_{t}, \quad t \in[0,1]
$$

and $d G_{1}=\phi_{1}$ on $\left.T D\right|_{S}$.

Set $\widetilde{\theta}_{t, s}=\ell_{t} \oplus \theta_{t, s}:\left.\left.T D\right|_{S} \hookrightarrow T X\right|_{g_{t}(S)}$ for $t, s \in[0,1]$ (a real vector bundle monomorphism over $g_{t}$ ). From the above properties (i)-(v) of $\theta_{t, s}$ we obtain

(i') $\widetilde{\theta}_{t, 0}=\ell_{t} \oplus d g_{t}=\left.d G_{t}\right|_{\left.T D\right|_{S}}$ (bottom side),

(ii') $\widetilde{\theta}_{t, 1}=\left.\ell_{t} \oplus \psi_{t}\right|_{T S}=\left.\phi_{t}\right|_{\left.T D\right|_{S}}$ (top side),

(iii') $\widetilde{\theta}_{0, s}=\left.\iota_{s}\right|_{\left.T D\right|_{S}}$ (left side),

(iv') $\widetilde{\theta}_{1, s}=\ell_{1} \oplus d g_{1}=\left.d G_{1}\right|_{\left.T D\right|_{S}}$ (right side), and

(v') $\widetilde{\theta}_{t, s}$ covers $g_{t}$ for every $t, s \in[0,1]$.

We wish to extend the monomorphisms $\widetilde{\theta}_{t, s}:\left.\left.T D\right|_{S} \hookrightarrow T X\right|_{g_{t}(S)}$ to real vector bundle monomorphisms $\Theta_{t, s}: T D \rightarrow T X(t, s \in[0,1])$ covering the immersions $G_{t}: D \hookrightarrow X$. Such extension already exists for $(t, s)$ in the bottom, top and left face of the parameter square $[0,1]^{2}$ where we respectively take $d G_{t},\left.\phi_{t}\right|_{T D}$ and $\iota_{s}$ (properties (i'), (ii') and (iii')). The homotopy lifting property provides an extension $\Theta_{t, s}$ for all $(t, s) \in[0,1]^{2}$, with the given boundary values on the bottom, top and left side of $[0,1]^{2}$. (See Fig. 4 , the front and the back face belong to the homotopy $\widetilde{\theta}_{t, s}$ over $S=\partial D$, compare with Fig. 3.) Over the right face $\{t=1\}$ we thus obtain a homotopy $\Theta_{1, s} \in \operatorname{Hom}_{i n j}\left(T D,\left.T X\right|_{G_{1}(D)}\right)(s \in[0,1])$ satisfying

$$
\Theta_{1,0}=d G_{1}:\left.T D \rightarrow T X\right|_{G_{1}(D)}, \quad \Theta_{1,1}=\left.\phi_{1}\right|_{T D}:\left.T D \rightarrow T X\right|_{G_{1}(D)} .
$$

The homotopy $\Theta_{1, s}$ is fixed over $S$ where it coincides with $\widetilde{\theta}_{1, s}=\left.d G_{1}\right|_{\left.T D\right|_{S}}$ by property (iv'). (On Fig. $4, \Theta_{1, s}$ appears on the right face of the cube, 
with bold vertical sides indicating that it is constant on $\left.T D\right|_{S}$ where it equals $\left.\ell_{1} \oplus d g_{1}.\right)$

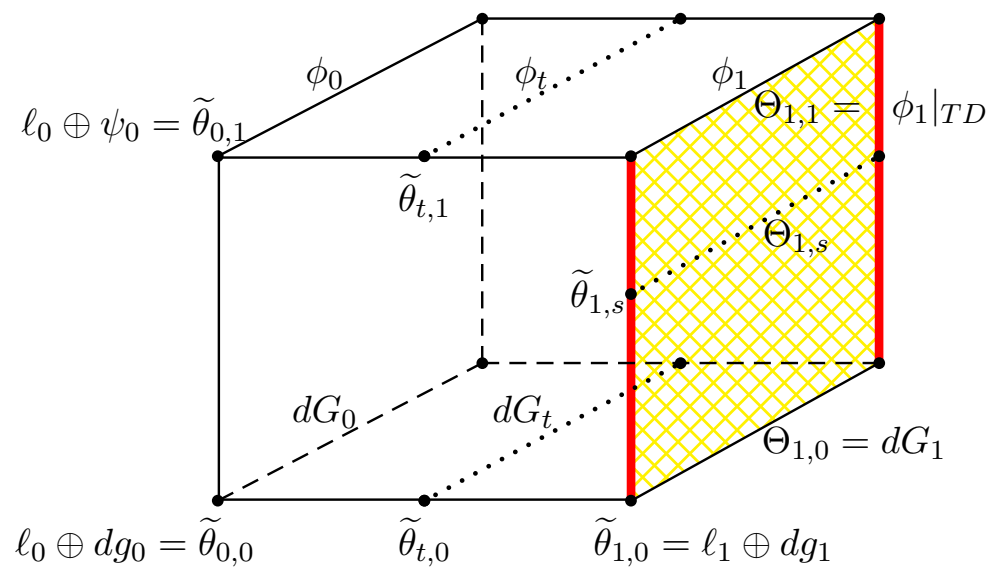

Figure 4. The homotopy $\Theta_{t, s}$

Since $\phi_{1}:\left.\left.T \mathbb{C}^{n}\right|_{D} \rightarrow T X\right|_{G_{1}(D)}$ is a $\mathbb{C}$-linear vector bundle isomorphism, the h-principle for totally real immersions (see [11, 35, 36]) provides a regular homotopy of immersions $G_{t}: D \rightarrow X \backslash W(t \in[1,2])$, fixed near $S$, such that the immersion $G_{2}$ is $J$-real and its complexified differential $d^{\mathbb{C}} G_{2}$ is homotopic to $\phi_{1}$ in the space of $\mathbb{C}$-linear maps $\left.T \mathbb{C}^{n}\right|_{D} \rightarrow T X$ of maximal rank. If in addition $G_{1}$ is an embedding, we can deform it to a totally real embedding $G_{2}$ by an isotopy which is fixed near $S$; this follows from the fact that totally real embeddings also satisfy the h-principle (see [36]). For $k<n$ or $k=n>2$ this can also be seen from the results in [14], and for $k=n=2$ it follows from the work of Eliashberg and Harlamov [10] on cancellation of complex points of real surfaces in complex surfaces (this is discussed in $\S 7$ below; see also [15]).

Finally we reparametrize the family $\left\{G_{t}: t \in[0,2]\right\}$ back to the parameter interval $[0,1]$. This proves the existence of a regular homotopy with the required properties.

It remains to be seen that, unless $k=n=2$, there also exists an isotopy of embeddings $\left\{G_{t}\right\}$ with these properties. If $k<n$, a small perturbation of $\left\{g_{t}\right\}$ with fixed ends at $t=0,1$ gives an isotopy which can be realized by an ambient diffeotopy, and we get an isotopy of embedded discs $G_{t}: D \hookrightarrow X \backslash W$ with $\left.G_{t}\right|_{S}=g_{t}$. For $k=n=1$ the conclusion of Lemma 3.1 obviously holds for any attached 1-disc (segment).

In the remainder of the proof we consider the case $k=n>2$. The main idea of the following argument was communicated to us by Y. Eliashberg (personal communication, June 2005). 
A generic choice of the regular homotopy $g_{t}: S \simeq S^{n-1} \rightarrow \Sigma$ insures that $g_{t}$ is an embedding for all but finitely many parameter values $t \in[0,1]$, and it has a simple (transverse) double point at each of the exceptional parameter values. We wish to change the Legendrian embedding $g_{1}$ by a regular homotopy of Legendrian immersions $g_{t}: S \hookrightarrow \Sigma(t \in[1,2])$ to another Legendrian embedding $g_{2}$ so that the resulting regular homotopy $\left\{g_{t}: t \in[0,2]\right\}$ will have self-intersection index zero. More precisely, the map $\widetilde{g}: \widetilde{S}=S \times[0,2] \rightarrow \widetilde{\Sigma}=\Sigma \times[0,2]$, defined by $\widetilde{g}(x, t)=\left(g_{t}(x), t\right)$, is an immersion of the $n$-dimensional oriented manifold $\widetilde{S}$ into the $2 n$-dimensional oriented manifold $\widetilde{\Sigma}$ such that the double points of $\widetilde{g}$ correspond to the double points of the regular homotopy $\left\{g_{t}\right\}$, and we define the index $i\left(\left\{g_{t}\right\}\right)$ as the number of double points of $\widetilde{g}$, counted with their orientation signs.

If this index equals zero then a foliated version of the Whitney trick allows us to deform $\left\{g_{t}\right\}_{t \in[0,2]}$ with fixed ends to an isotopy of embeddings. This is done by connecting a chosen pair of double points $q_{0}, q_{1} \in \widetilde{g}(\widetilde{S})$ of the opposite sign, lying over two different values $t_{0}<t_{1}$ of the parameter, by a pair of curves $\lambda_{j}(t)=\widetilde{g}\left(c_{j}(t), t\right)\left(t \in\left[t_{0}, t_{1}\right], j=1,2\right)$ which together bound an embedded Whitney disc $D^{2} \subset \widetilde{\Sigma}$ such that $D^{2} \cap\left(\Sigma_{t} \times\{t\}\right)$ is an arc connecting $\lambda_{1}(t)$ to $\lambda_{2}(t)$ for every $t \in\left[t_{0}, t_{1}\right]$, and it degenerates to $q_{0}$ resp. $q_{1}$ over the endpoints $t_{0}$ resp. $t_{1}$. The rest of the procedure, removing this pair of double points by pulling $\widetilde{g}(\widetilde{S})$ across $D^{2}$, is standard [60]. Performing this operation finitely many times one can remove all double points and change $\left\{g_{t}\right\}$ to an isotopy of embeddings.

The rest of the proof can be completed exactly as before: we extend $g_{t}$ to an isotopy of embedded discs $G_{t}: D \hookrightarrow X \backslash W$, with $\left.G_{t}\right|_{S}=g_{t}$, covered by a homotopy of $\mathbb{C}$-linear isomorhisms $\phi_{t}:\left.\left.T \mathbb{C}^{n}\right|_{D} \rightarrow T X\right|_{G_{t}(D)}$. Observe that $\left\{d g_{t}\right\}$ still has the correct homotopy property so that the final embedding $G_{2}$ can be deformed (with fixed boundary) to a totally real embedding.

It remains to see that the index of $\left\{g_{t}\right\}_{t \in[0,1]}$ can be changed to an arbitrary number (in particular, to zero) by a small Legendrian deformation of $g_{1}$ in $\Sigma$. This will be realized by a local Legendrian isotopy which introduces the correct number of double points. (A similar deformation is used in [7, $\S 2.4]$ for changing a stably special HAT to a special one.)

Set $L=L_{1}:=g_{1}(S) \subset \Sigma$, an embedded Legendrian sphere. Choose a point $a \in L \subset \Sigma$. In suitable local coordinates $(z, q, p) \in \mathbb{R}^{2 n-1}$ on $\Sigma$, with $a$ corresponding to $0 \in \mathbb{R}^{2 n-1}$, the contact form is $\eta=d z-\sum_{j=1}^{n-1} p_{j} d q_{j}$, and $L$ is given by the equations

$$
\left\{(z, q, p) \in \mathbb{R}^{2 n-1}: z^{2}=q_{1}^{3}, p_{1}^{2}=\frac{9}{4} q_{1}, p_{2}=\cdots=p_{n-1}=0\right\} .
$$

(Section 2.4 in [7].) Let $\pi: \mathbb{R}^{2 n-1} \rightarrow \mathbb{R}^{n-1}$ denote the projection $\pi(z, q, p)=$ $q$. Choose a closed ball $\Delta \subset \mathbb{R}^{n-1}$ centered at $\left(q_{1}^{0}, 0, \ldots, 0\right)$ for a small $q_{1}^{0}>0$, of radius $q_{1}^{0} / 2$. Let $\phi: \Delta \rightarrow \mathbb{R}$ be a smooth function equaling 0 near 
$\partial \Delta$. Set

$$
h_{t}(q)=q_{1}^{3 / 2}(1+(t-1) \phi(q)), \quad t \in[1,2] .
$$

Let $L_{t}$ equal $L$ outside of $\pi^{-1}(\Delta)$ and equal

$$
\left\{(z, q, p): z=h_{t}(q), p=\frac{\partial h_{t}}{\partial q}(q)\right\} \cup\left\{(z, q, p): z=-h_{t}(q), p=-\frac{\partial h_{t}}{\partial q}(q)\right\}
$$

over $\Delta$. (We choose $\phi$ with sufficiently small derivative to insure that we remain in the given coordinate patch; this can be done if $q_{1}^{0}>0$ is chosen small enough.) Let $g_{t}: S \rightarrow \Sigma(t \in[1,2])$ be the regular homotopy such that $g_{t}(S)=L_{t}$. The deformation is illustrated by Fig. [5. The top diagrams show the projection onto the $(z, q)$-plane at three typical stages, with the cusp at $(z, q)=(0,0)$ and with a self-intersection shown in the middle figure.

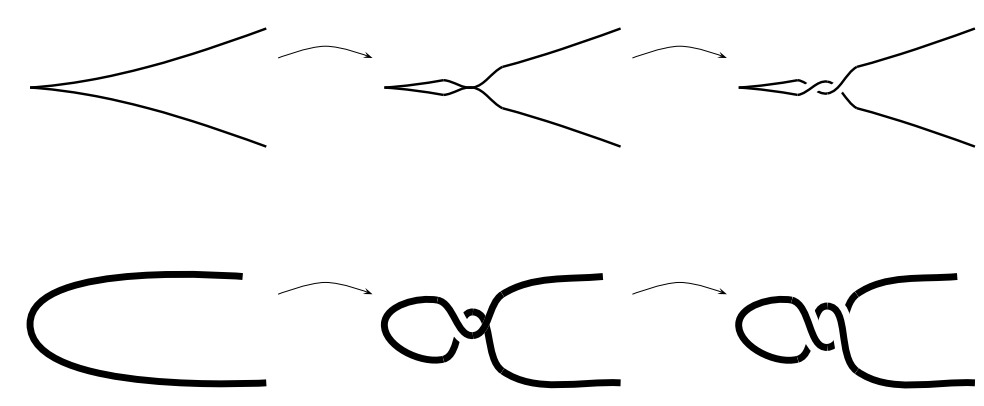

Figure 5. Changing the index of a regular homotopy by +1 .

The index of $\left\{g_{t}\right\}_{t \in[1,2]}$ equals the intersection number between the manifolds

$$
M_{ \pm}=\left\{(z, q, p, t) \in \mathbb{R}^{2 n}: z= \pm h_{t}(q), p= \pm \frac{\partial h_{t}}{\partial q}(q), q \in \Delta, t \in[1,2]\right\} .
$$

The intersection points of $M_{+}$and $M_{-}$are solutions of the equations

$$
1+(t-1) \phi=0, \quad \frac{\partial \phi}{\partial q}=0, \quad t \in[1,2] .
$$

This is precisely the set of all critical points of $\phi$ with the critical values belonging to $(-\infty,-1]$. By a generic choice of $\phi$ we can insure that -1 is not a critical value of $\phi$. A computation shows that each point $(q, t)$ satisfying the above equations adds \pm 1 to the index $i\left(\left\{g_{t}\right\}\right)$, depending on the sign of the determinant of the Hessian Hess $(\phi)$ at $q$; hence we get +1 at a critical point of even Morse index and -1 at a critical point of odd Morse index. Similarly, as we increase $c \in \mathbb{R}$, the Euler characteristic of the sublevel set $\{\phi \leq c\}$ increases by one at every critical point of $\phi$ of even Morse index, and it decreases by one at every critical point of odd Morse index. We conclude that $i=i\left(\left\{g_{t}\right\}_{t \in[1,2]}\right)$ equals the Euler number of the set $\{q \in \Delta: \phi(q) \leq-1\}$. If $n>2$, this can be arranged to equal any preassigned 
integer by a suitable choice of $\phi$, and hence we can arrange the index $i$ to equal zero. If $n=2$ then $i$ can be arranged to be any nonnegative number since $\{\phi \leq-1\}$ is a union of segments, but it cannot be negative.

This completes the proof of Lemma 3.1 in the smooth case. Assume now that $J$ is integrable in a neighborhood of the disc $G_{0}(D)$ and the hypersurface $\Sigma=\partial W$ is real analytic near the attaching sphere $G_{0}(S) \subset \Sigma$ with respect to the induced complex structure; we wish to find a real analytic disc satisfying the conclusion of Lemma 3.1. Since the disc $G_{1}$ constructed above can be chosen arbitrarily $\mathcal{C}^{0}$-close to $G_{0}$, we may assume that the same conditions on $J$ and $\Sigma$ also hold near $G_{1}(D)$. By [7, Lemma 2.5.1.] (which uses Gray's theorem on real analytic approximation of Legendrian embeddings) it is possible to approximate $G_{1}$ in the $\mathcal{C}^{1}$ topology by a disc $G_{1}^{\prime}:(D, S) \rightarrow$ $(X \backslash W, \Sigma)$ which is real analytic near $S$ such that $\left.G_{1}^{\prime}\right|_{S}: S \hookrightarrow \Sigma$ is Legendrian and $G_{1}^{\prime}$ is normal to $\Sigma$ along $S$. It remains to perturb $G_{1}^{\prime}$ to a nearby real analytic map $G_{1}^{\prime \prime}: D \rightarrow X$ which agrees with $G_{1}^{\prime}$ to the second order along $S$ and to replace $G_{1}$ by $G_{1}^{\prime \prime}$.

Remark 3.2. We wish to point out that in the critical case $k=n>2$ Lemma 3.1 does not seem a direct consequence of the results in 7]. Let $D=D^{n}$ and $S=\partial D$. In [7, $\left.\S 2\right]$ it is shown that for $n \neq 2$ every embedding $G:(D, S) \rightarrow(X \backslash W, \Sigma)$ can be isotoped to one for which $G$ is totally real near the boundary, the attaching sphere $G(S)$ is Legendrian in $\Sigma$, and the associated normal framing $\beta:\left.\nu \rightarrow T X\right|_{G(D)}$ satisfies

$$
\beta \circ J_{s t}=J \circ d G \text { on } T_{x} D, \quad x \in S .
$$

This means that the triple $(G, \beta, \phi=d G \oplus \beta)$, restricted to the points of $S$, is a special $H A T$ in the sense of 7, p. 33]. Choose a diffeomorphism $\widetilde{G}$ from a standard handle $H \subset \mathbb{C}^{n}$ onto $\widetilde{G}(H) \subset X$ such that $\left.\widetilde{G}\right|_{D}=G$ and $\left.d \widetilde{G}\right|_{\nu}=\beta$. By (3.1) the push-forward $\widetilde{J}=\widetilde{G}_{*}\left(J_{s t}\right)$ of the standard complex structure on $\mathbb{C}^{n}$ agrees with $J$ at every point of $G(S)$, and by a small correction these structures can be made to agree in a neighborhood of $G(S)$ in $X$. Using the h-principle and some homotopy theory one can see that the disc $G$ (which is clearly $\widetilde{J}$-real) is isotopic to a $J$-real disc by an isotopy which is fixed near $S$ precisely when $\widetilde{J}$ is homotopic to $J$ along $G(D)$ by a homotopy which is fixed near $G(S)$. The latter condition holds if and only if the topological invariant in (3.2) below vanishes. The following proposition shows that this always holds for certain values of $n$ modulo 8 , and the proof of Lemma 3.1 avoids this potential problem in every dimension.

Proposition 3.3. Let $W$ be an open, relatively compact domain with smooth strongly pseudoconvex boundary $\Sigma=\partial W$ in an almost complex manifold $(X, J)$ of real dimension $2 n$. Let $D=D^{n}, S=\partial D$, and let $G:(D, S) \rightarrow$ $(X \backslash W, \Sigma)$ be a smooth embedding, normal to $\Sigma$, such that $G(S)$ is Legendrian in $\Sigma$. Assume that $G$ admits a normal framing $\beta$ satisfying (3.1) over $S$. If $n \in\{1,3,4,5\}$ modulo 8 then $G$ is isotopic to a totally real embedding by an isotopy which is fixed near $S$. 
Proof. We extend $G$ to a diffeomorphism $H \rightarrow G(H) \subset X$ satisfying $\left.d G\right|_{\nu}=$ $\beta$. The difference on $D$ between the almost complex structures $J^{\prime}=\widetilde{G}^{*} J$ and $J_{s t}$ (which agree over $S$ ) defines an element

$$
\delta\left(J^{\prime}, J_{s t}\right) \in\left[S^{n}, G L_{2 n}^{+}(\mathbb{R}) / G L_{n}(\mathbb{C})\right]=\pi_{n}(S O(2 n) / U(n))
$$

(Milnor [46, p. 133]). Using the long exact sequence of homotopy groups and the five lemma one sees that

$$
\pi_{n}(S O(2 n) / U(n))=\pi_{n}(S O / U)=\pi_{n}(\Omega S O)=\pi_{n+1}(S O) .
$$

By the (real) Bott periodicity theorem this group equals $\mathbb{Z}$ if $n \in\{2,6\}$ modulo 8, it equals $\mathbb{Z}_{2}$ if $n \in\{0,7\}$ modulo 8 , and it vanishes for the remaining values $n \in\{1,3,4,5\}$ modulo 8 . In the last case we conclude that $J=J_{1}$ is homotopic along $G(D)$ to $J_{0}=G_{*}\left(J_{s t}\right)$ by a homotopy which is fixed near $G(S) \subset \Sigma$. Since $G_{0}=G$ is clearly $J_{0}$-real, Gromov's h-principle for totally real embeddings [11, 14, 36] gives an isotopy of embedded discs $G_{t}:(D, S) \rightarrow(X \backslash W, \Sigma)$ which is fixed near $S$ such that $G_{t}$ is $J_{t}$-real for every $t \in[0,1]$. At $t=1$ we get a $J$-real embedded disc $G_{1}$.

\section{A holomorphic APPRoximation theOREM}

Let $X$ be a complex manifold with an integrable complex structure $J$. We denote by $\mathcal{H}(X)=\mathcal{H}(X, J)$ the algebra of all holomorphic functions on $X$. A compact set $K$ in $X$ is $\mathcal{H}(X)$-convex (or $\mathcal{H}(X, J)$-convex) if for every point $p \in X \backslash K$ there exists an $f \in \mathcal{H}(X)$ with $|f(p)|>\sup _{x \in K}|f(x)|$.

We say that a compact set $K$ in $X$ is holomorphically convex if there is an open Stein domain $\Omega \subset X$ containing $K$ such that $K$ is $\mathcal{H}(\Omega)$-convex. By the classical theory [40, Chapter 2] this is equivalent to the existence of a Stein neighborhood $\Omega$ of $K$ and a continuous plurisubharmonic function $\rho \geq 0$ on $\Omega$ such that $\rho^{-1}(0)=K$ and $\rho$ is strongly plurisubharmonic on $\Omega \backslash K$. We may take $\Omega=\left\{\rho<c_{1}\right\}$ for some $c_{1}>0$; for any $c \in\left(0, c_{1}\right)$ the sublevel set $\{\rho<c\} \Subset \Omega$ is then Stein and Runge in $\Omega$ [40, $\S 4.3]$.

A point $p_{0}$ in an immersed real $k$-dimensional submanifold $M \subset X$ is said to be a special double point if there is a holomorphic coordinate system

$$
z=\left(x^{\prime}+i y^{\prime}, z^{\prime \prime}\right): U \rightarrow \widetilde{U} \subset \mathbb{C}^{n}=\mathbb{R}^{k} \oplus i \mathbb{R}^{k} \oplus \mathbb{C}^{n-k}
$$

in a neighborhood $U \subset X$ of $p_{0}$ such that $z\left(p_{0}\right)=0$ and

$$
z(M \cap U)=\widetilde{U} \cap\left(\left\{\left(x^{\prime}+i 0^{\prime}, 0^{\prime \prime}\right): x^{\prime} \in \mathbb{R}^{k}\right\} \cup\left\{\left(0^{\prime}+i y^{\prime}, 0^{\prime \prime}\right): y^{\prime} \in \mathbb{R}^{k}\right\}\right)
$$

The following approximation theorem will be used in the proof of Lemma 5.1. It is far from the most general one with respect to the type of allowed double points of $M$, but it will suffice for our purposes.

Theorem 4.1. Let $K_{0}$ and $M$ be compact sets in a complex manifold $X$, where $M \backslash K_{0}$ is a smoothly immersed totally real submanifold such that each 
non-smooth point is a special double point (4.1). Assume that $K_{0}$ is holomorphically convex and there is a compact holomorphically convex relative neighborhood $N$ of $K_{0}$ in $K:=K_{0} \cup M$ (Fig. 6).

Given a continuous map $f: X \rightarrow Y$ to a complex manifold $Y$ such that $f$ is holomorphic in an open neighborhood of $K_{0}$, there exist open Stein domains $V_{1} \supset V_{2} \supset \cdots \supset \cap_{j} V_{j}=K$ and holomorphic maps $f_{j}: V_{j} \rightarrow Y$ $(j=1,2, \ldots)$ such that $K_{0}$ and $K$ are $\mathcal{H}\left(V_{j}\right)$-convex for every $j$, and $\left.f_{j}\right|_{K} \rightarrow$ $\left.f\right|_{K}$ uniformly as $j \rightarrow \infty$. If $M \backslash K_{0}$ is an embedded submanifold and $\left.f\right|_{M \backslash K_{0}}$ is of class $\mathcal{C}^{r}$ then $f_{j}$ can be chosen such that $\left.\left.f_{j}\right|_{K} \rightarrow f\right|_{K}$ uniformly and $\left.\left.f_{j}\right|_{M \backslash K_{0}} \rightarrow f\right|_{M \backslash K_{0}}$ in the $\mathcal{C}^{r}(M)$ topology as $j \rightarrow \infty$.

The analogous results hold for a family of maps $f: X \times P \rightarrow Y$ parametrized by a compact Hausdorff space $P$.

In the case when $M \backslash K_{0}$ is an embedded totally real submanifold this is essentially Theorem 3.2 in 18 . Uniform approximation was obtained by Hörmander and Wermer [41] in the case $X=\mathbb{C}^{n}, Y=\mathbb{C}$ and $M \backslash K_{0}$ a totally real embedded $\mathcal{C}^{1}$ submanifold. Another special case is due to Fornæss and Stout [12].

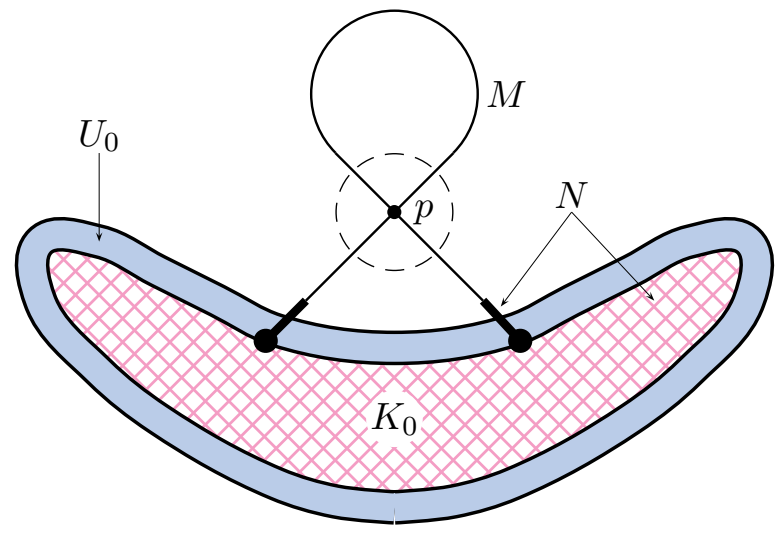

Figure 6. A kinky disc $M$ attached to $K_{0}$

Proof. Consider first the case when $M \backslash K_{0}$ is an embedded totally real submanifold. The assumption regarding $N$ implies by [18, Theorem 3.1] that the set $K=K_{0} \cup M$ is holomorphically convex. (See also [41] for the case $X=\mathbb{C}^{n}$.) More precisely, given an open neighborhood $U_{0} \subset X$ of $K_{0}$, there exists a continuous plurisubharmonic function $\rho \geq 0$ in an open neighborhood $U \subset X$ of $K$ such that $\rho$ is smooth strongly plurisubharmonic on $U \backslash U_{0}$ and $\rho^{-1}(0)=K$ [18, p. 1923]. Taking $V_{j}=\left\{x \in U: \rho(x)<c_{j}\right\}$ for a decreasing sequence of small numbers $c_{j}>0$ converging to zero we get a Stein neighborhood basis of $K$ such that $K$ is $\mathcal{H}\left(V_{j}\right)$-convex for each $j$. It is easily 
seen that $K_{0}$ is also $\mathcal{H}\left(V_{j}\right)$-convex. Indeed, choose a smooth function $\chi \geq 0$ with compact support in $U$ which vanishes on an open set $U_{1} \supset \bar{U}_{0}$ and is positive on $M \backslash \bar{U}_{1}$. Then the function $\rho+\epsilon \chi \geq 0$ is still plurisubharmonic for sufficiently small $\epsilon>0$ and hence its zero set $K \cap \bar{U}_{1}$ is $\mathcal{H}(K)$-convex (i.e., for every point $p \in K \backslash \bar{U}_{1}$ there is a holomorphic function $g$ in a neighborhood of $K$ with $\left.1=g(p)>\sup _{x \in K \cap \bar{U}_{1}}|g(x)|\right)$. Since $U_{0}$ and $U_{1}$ can be chosen arbitrary close to $K_{0}$, we see that $K_{0}$ is $\mathcal{H}(K)$-convex, and hence also $\mathcal{H}\left(V_{j}\right)$-convex.

By approximation we may assume that $f$ is smooth on $M \backslash K_{0}$. Theorem 3.2 in [18] now shows that $f$ can be approximated uniformly on $K_{0}$, an in the $\mathcal{C}^{r}$ topology on $M \backslash K_{0}$, by maps which are holomorphic in small open neighborhoods of $K$ in $X$ (the size of the neighborhood depends on the rate of approximation). Choosing these neighborhoods from the above sequence $V_{j}$ we get the conclusion of Theorem 4.1,

Suppose now that $M \backslash K_{0}$ is an immersed totally real submanifold with special double points $p_{1}, \ldots, p_{m}$. (Replacing $K_{0}$ by a relative neighborhood of $K_{0}$ in $K$ we can assume that there are only finitely many such points.) Let $B_{j} \subset X \backslash K_{0}$ be a small open neighborhood of $p_{j}$ in $X$ such that $\bar{B}_{j}$ is mapped onto a closed ball around $0 \in \mathbb{C}^{n}$ by a local coordinate map (4.1). By a uniformly small change we make $f$ smooth on $M \backslash K_{0}$ and constantly equal to $f\left(p_{j}\right)$ on a neighborhood of $\bar{B}_{j}$ in $X$; the latter change can be made small by choosing the balls $B_{j}$ as small as necessary. We can now apply the previous argument with the set $K_{0}^{\prime}=K_{0} \cup\left(\cup_{j=1}^{m} \bar{B}_{j}\right)$ and the embedded totally real submanifold $M^{\prime}=M \backslash K_{0}^{\prime}$. Indeed, $K_{0}^{\prime}$ is clearly holomorphically convex, and it has a compact holomorphically convex relative neighborhood $N^{\prime}$ in $K^{\prime}:=K_{0}^{\prime} \cup M^{\prime}=K \cup\left(\cup_{j=1}^{m} \bar{B}_{j}\right)$. (It suffices to take $N^{\prime}=N \cup\left(\cup_{j=1}^{m} N_{j}\right.$ ) where $N_{j}$ is the union of $\bar{B}_{j}$ with a suitably chosen small collar in $M$; in local coordinates, $N_{j}$ corresponds to the union of a closed ball in $\mathbb{C}^{n}$ centered at 0 with a collar in $\mathbb{R}^{k} \cup i \mathbb{R}^{k} \subset \mathbb{C}^{k} \times\{0\}^{n-k}$.) As before, Theorem 3.1 in [18] implies that $K^{\prime}$ is holomorphically convex, and Theorem 3.2 in [18] gives a desired approximation of $f$ by holomorphic maps in small Stein neighborhoods of $K^{\prime}$ in $X$.

The proof of Theorem 4.1 for a family of maps $f: X \times P \rightarrow Y$, with $P$ a compact Hausdorff space, is obtained by covering the graph of the family in $X \times Y$ (after an initial smoothing of the maps $f(\cdot, p): X \rightarrow Y$ on the $M \backslash K_{0}$ ) by finitely many Stein neighborhoods in $X \times Y$, using these to approximate $f$ by local (in $P$ ) families of holomorphic maps, and patching these families by a continuous partition of unity in the parameter $p \in P$. The latter is possible since we can introduce a complex linear structure on the fibers of the projection $X \times Y \rightarrow X$ within a small Stein neighborhood of each individual graph. The details in a very similar context can be found in [23] (proof of Theorem 4.2, pp. 138-139). 


\section{EXTENDing A HOLOMORPHIC MAP ACROSS A HANDLE}

The following lemma is the key ingredient in the proofs of our main results.

Lemma 5.1. Let $(X, J)$ be an almost complex manifold of real dimension $2 n$. Let $W \Subset X$ be a smoothly bounded domain such that $J$ is integrable in a neighborhood of $\bar{W}$, the manifold $(W, J)$ is Stein, and $\Sigma=\partial W$ is strongly $J$-pseudoconvex. Let $D=D^{k}$ and $S=S^{k-1}=\partial D(1 \leq k \leq n)$. Let $G:(D, S) \rightarrow(X \backslash W, \Sigma)$ be a smooth $J$-real embedding which is normal to $\Sigma$ and such that $\left.G\right|_{S}: S \rightarrow \Sigma$ is Legendrian. Assume that $Y$ is a complex manifold and $f: X \rightarrow Y$ is a continuous map which is $J$-holomorphic in an open neighborhood of $\bar{W}$. Let $d_{Y}$ be a distance function on $Y$ induced by a smooth Riemannian metric.

After a small smooth perturbation of $G$ there exist an integrable complex structure $\widetilde{J}$ in an open neighborhood $U \subset X$ of $K:=\bar{W} \cup G(D)$, a homotopy $J_{t}(t \in[0,1])$ of almost complex structures on $X$ which is fixed on a neighborhood of $\bar{W}$ and on $M=G(D)$, with $J_{0}=J$ and $J_{1}=\widetilde{J}$, and for every $\epsilon>0$ there exist a smoothly bounded strongly $\widetilde{J}$-pseudoconvex Stein domain $\widetilde{W}$ and a map $\widetilde{f}: X \rightarrow Y$ satisfying the following:

(i) $\bar{W} \cup G(D) \subset \widetilde{W} \subset U, \widetilde{W}$ is a handlebody with core $K=\bar{W} \cup G(D)$, and $\bar{W}$ is $\mathcal{H}(\widetilde{W}, \widetilde{J})$-convex.

(ii) The map $\left.\widetilde{f}\right|_{\widetilde{W}}: \widetilde{W} \rightarrow Y$ is $\widetilde{J}$-holomorphic.

(iii) There is a homotopy $f_{t}: X \rightarrow Y(t \in[0,1])$, with $f_{0}=f$ and $f_{1}=$ $\tilde{f}$, such that for each $t \in[0,1]$ the map $f_{t}$ is J-holomorphic on a neighborhood of $\bar{W}$ and $\sup _{x \in \bar{W}} d\left(f(x), f_{t}(x)\right)<\epsilon$.

If in addition $f$ is covered by a complex vector bundle map $\iota:(T X, J) \rightarrow$ $T Y$ which is of maximal rank on every fiber and such that $d f=\iota$ on a neighborhood of $\bar{W}$ then we can choose $\widetilde{f}$ to be of maximal rank at every point of $\widetilde{W}$ and such that $d \widetilde{f}$ is homotopic to $\iota$ through complex vector bundle maps $\iota_{t}:\left(T X, J_{t}\right) \rightarrow T Y$ of pointwise maximal rank.

If $\Sigma$ is real analytic and the almost complex structure $J$ is integrable in a neighborhood of $K$ then the above conclusions hold with $J=\widetilde{J}$.

The analogous results hold for a continuous family of maps with a parameter in a compact Hausdorff space.

Proof. After a small enlargement of $W$ and a small deformation of $G$ we may assume that $\partial W$ is real analytic and strongly $J$-pseudoconvex, $J$ is integrable in a neighborhood of $\bar{W}$, and the $k$-disc $M:=G(D)$ is attached to $\bar{W}$ along the Legendrian $(k-1)$-sphere $G(S) \subset \partial W$. By [7, Lemma 2.5.1.] (which uses Gray's theorem on approximation of Legendrian embeddings by real analytic Legendrian embeddings) we can approximate $G$ by a map which is normal to $\Sigma$ and real analytic in a neighborhood of $S=\partial D$, such that the attaching sphere $G(S) \subset \Sigma$ is Legendrian in $\Sigma=\partial W$. 
We first consider the case $k=n$. For every $x \in D$ let $A_{x}: T_{x} \mathbb{C}^{n} \rightarrow T_{G(x)} X$ denote the unique $\left(J_{s t}, J\right)$-linear map which agrees with $d G_{x}$ on $T_{x} D$. We extend $G$ to a smooth diffeomorphisms $\widetilde{G}$ from a standard handle $H \subset \mathbb{C}^{n}$ (2.1) onto a neighborhood $\widetilde{H}=\widetilde{G}(H)$ of $G(D)$ in $X$ such that $d \widetilde{G}_{x}=A_{x}$ for each $x \in D$. Near the sphere $S=\partial D$ we take $\widetilde{G}$ to be the complexification of $G$, hence biholomorphic. If $J$ is integrable then we can choose $G$ to be real analytic and $\widetilde{G}$ to be its complexification (Lemma 3.1).

Let $W^{\prime}$ be a slightly larger domain in $X$ containing $\bar{W}$. Let $\widetilde{J}$ denote the complex structure on $W_{1}:=W^{\prime} \cup \widetilde{H}$ which equals $J$ on $W^{\prime}$ and equals $\widetilde{G}_{*}\left(J_{s t}\right)$ on the handle $\widetilde{H}$. By choosing the sets $W^{\prime} \supset \bar{W}$ and $H \supset D$ sufficiently small we insure that these two complex structures coincide on $W^{\prime} \cap \widetilde{H}$ (since $\widetilde{G}$ maps a neighborhood of $S \subset \mathbb{C}^{n}$ biholomorphically onto a neighborhood of $G(S) \subset X)$. Notice also that $J=\widetilde{J}$ at every point of $M=G(D)$ since $d \widetilde{G}_{x}=A_{x}$ was chosen to be $\left(J_{s t}, J\right)$-linear for each $x \in D$. This clearly implies the existence of a homotopy of almost complex structures $\left\{J_{t}\right\}_{t \in[0,1]}$ on $W_{1}$ which is fixed on $W^{\prime} \cup M$ and satisfies $J_{0}=J, J_{1}=\widetilde{J}$. If $G$ is real analytic then $J=\widetilde{J}$ near $M$ and we can choose $J_{t}=J$ for all $t \in[0,1]$.

Our next goal is to (approximately) extend $f: X \rightarrow Y$ to a holomorphic map across the handle. By the assumption $f$ is $J$-holomorphic in a neighborhood of $\bar{W}$ in $X$, and we may assume by approximation that it is smooth on $X$. Since $\widetilde{J}=J$ near $\bar{W}, f$ is also $\widetilde{J}$-holomorphic near $\bar{W}$. We wish to apply Theorem 4.1 in the complex manifold $\left(W_{1}, \widetilde{J}\right)$, with the compact sets $K_{0}=\bar{W}$ and $K=K_{0} \cup M$, in order to obtain a $\widetilde{J}$-holomorphic map $\tilde{f}: V \rightarrow Y$ in an open neighborhood $V \supset K$ such that $\left.\widetilde{f}\right|_{K}$ approximates $\left.f\right|_{K}$ as close as desired. In order to do so, we must verify that $\bar{W}$ has a compact holomorphically convex relative neighborhood $N$ in $K$. It is well known (see e.g. [52, Lemma 1]) that the problem is local near the attaching sphere $G(S)=\partial M \subset \Sigma$. Thus, taking a closed tubular neighborhood $T \subset X$ of $M=G(D)$, it suffices to show that the set $\widetilde{G}^{-1}(T \cap K) \subset \mathbb{C}^{n}$ is holomorphically convex for a suitable choice of $T$. The latter set is the union of the closed disc $D \subset \mathbb{R}^{n} \subset \mathbb{C}^{n}$ and a piece of a strongly pseudoconvex domain which essentially looks like the quadric $Q_{\lambda}(2.2)$. In fact, by a small outward bumping of $\Sigma=\partial W$ (from the side of $W$ ) which is localized in a tubular neighborhood of the circle $G(S)$ (keeping $\Sigma$ and its tangent bundle fixed on $G(S))$ we can reduce to the situation when $\widetilde{G}^{-1}(T \cap K)=\widetilde{G}^{-1}(T) \cap\left(Q_{\lambda} \cup D\right)$ and $\widetilde{G}^{-1}(T)$ is a compact convex set in $\mathbb{C}^{n}$. The holomorphic polynomial $h(z)=z_{1}^{2}+\ldots+z_{n}^{2}$ on $\mathbb{C}^{n}$ maps the disc $D$ to the segment $[0,1] \subset \mathbb{R} \subset \mathbb{C}$, it maps the sphere $S=\partial D$ to the point 1 , and $\Re h>1$ on the set $Q_{\lambda} \backslash S$. (Compare with 52 and the proof of Lemma 6.6 in 17].) Thus $h$ separates the polynomially convex sets $Q_{\lambda} \cap \widetilde{G}^{-1}(T)$ and $D$, and hence their union is polynomially convex in $\mathbb{C}^{n}$ by a lemma of Eva Kallin [55, Lemma 29.21]. 
Thus Theorem 4.1 applies and gives a $\widetilde{J}$-holomorphic map $\widetilde{f}$ in a neighborhood of $K$ which approximates $f$ uniformly on $K$. A homotopy from $f$ to $\tilde{f}$ with the required properties clearly exists near $K$ provided that the approximation is sufficiently close, and it is then used to patch $\widetilde{f}$ with $f$ outside of a larger neighborhood of $K$.

Remaining in the case $k=n$ for the moment, we also consider the situation when $f$ is of maximal complex rank in a neighborhood of $\bar{W}$ and is covered by a complex vector bundle map $\iota:(T X, J) \rightarrow\left(T Y, J_{Y}\right)$ of fiberwise maximal rank, with $\iota=d f$ near $\bar{W}$. In this case we must show that $\widetilde{f}$ can also be chosen of maximal rank on $M$, and hence in a neighborhood of $K$ provided that the approximation is sufficiently close. To this end we first deform $f$ (without changing it near $\bar{W}$ ) such that for every $x \in M$ its differential $d f_{x}: T_{x} M \rightarrow T_{f(x)} Y$ is of maximal complex rank (equal to $\min \{n, \operatorname{dim} Y\})$, and the map $x \rightarrow d f_{x}(x \in M)$ is homotopic to $\left.x \rightarrow \iota\right|_{T_{x} M}$ by a homotopy of vector bundle maps of pointwise maximal complex rank which is fixed near $\partial M$. This is a straightforward application of Gromov's h-principle, the main point being that the pertinent differential relation is ample on any totally real submanifold. For details see [17, Lemma 6.4] or [18. Lemma 4.3, p. 1931]. Applying Theorem 4.1 to this new map $f$ we obtain a $\widetilde{J}$-holomorphic map $\widetilde{f}$ in a neighborhood of $K$ which approximates $f$ uniformly on $\bar{W}$ and in the $\mathcal{C}^{1}$-topology on $M$. If the approximation is sufficiently close, the latter property insures that $\tilde{f}$ is of maximal rank at every point of $K$. The existence of a homotopy from $\iota$ to $d \widetilde{f}$ with the required properties follows from the construction (see [17] for the details).

To complete the proof of Lemma 5.1 (still in the case $k=n$ ) it remains to find a $\widetilde{J}$-convex Stein domain $\widetilde{W} \supset K$ contained in $V$ (so that $\widetilde{f}$ will be holomorphic in $\widetilde{W}$ ) and satisfying the other required properties; see Fig. 7 , Assuming as we may that $\Sigma$ has been standardized along $G(S)$ as described above, this is an immediate application of Lemma 2.1 - one takes $\widetilde{W}=$ $\widetilde{G}(\operatorname{Int} L)$ where $L \subset \mathbb{C}^{n}$ is a standard strongly pseudoconvex handlebody around $Q_{\lambda} \cup D$ as in the cited lemma. Eliashberg also showed how to extend a $J$-convex defining function $\rho$ for $W$ to a $\widetilde{J}$-convex defining function $\widetilde{\rho}$ for $\widetilde{W}$ with precisely one additional Morse critical point of index $\operatorname{dim}_{\mathbb{R}} D$ which may be placed at the center of the attached handle. It follows in particular that $W$ and $\widetilde{W}$ are two sublevel sets of the same $\widetilde{J}$-convex exhaustion function and hence $W$ is Runge in $\widetilde{W}$. This also follows from our earlier argument on polynomial convexity of $Q_{\lambda} \cup D$.

This completes the proof of Lemma 5.1 for $k=n$. When $1 \leq k<n$, we apply the same proof with a totally real $n$-disc $M^{\prime}$ obtained by thickening $M=G(D)$ in the missing $n-k$ real directions. To find such $M^{\prime}$ we choose a $\mathbb{C}$-linear normal framing $\beta:\left.\nu^{\prime \prime} \rightarrow T X\right|_{M}$ over $G: D \hookrightarrow X$ such that $d^{\mathbb{C}} G \oplus$ $\beta: T^{\mathbb{C}} D \oplus \nu^{\prime \prime}=\left.\left.T \mathbb{C}^{n}\right|_{D} \rightarrow T X\right|_{G(D)}$ is a $\mathbb{C}$-linear isomorphism. Furthermore, we may choose $\beta$ to map $\left.\nu^{\prime \prime}\right|_{S}$ into the contact subbundle $\left.\xi\right|_{G(S)}$. Let $r D^{n-k}$ 


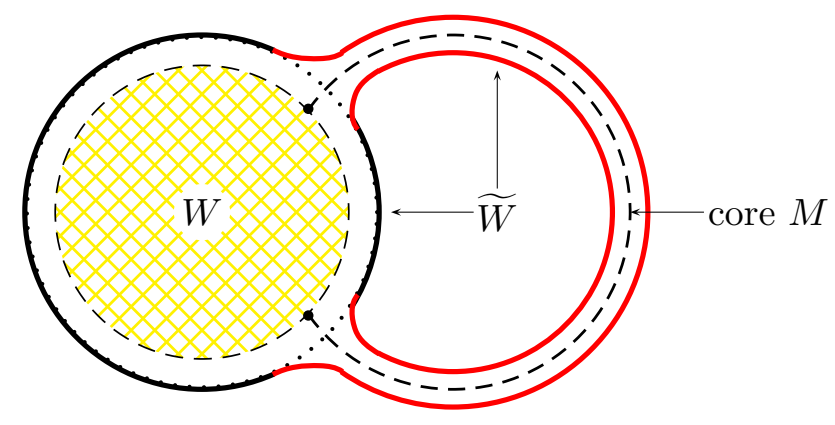

Figure 7 . The handlebody $\widetilde{W}$ around $K=\bar{W} \cup M$

denote a closed ball of radius $r>0$ in the real subspace $\{0\}^{k} \oplus\{i 0\}^{k} \oplus$ $\mathbb{R}^{n-k} \oplus\{i 0\}^{n-k} \subset \mathbb{C}^{n}$. For a small $r>0$ we can extend $G$ to a smooth $J$-real embedding $(1+r) D^{k} \times r D^{n-k} \hookrightarrow X$, still denoted $G$, which is real analytic near $S^{k-1} \times r D^{n-k}$, it maps the latter manifold to a Legendrian submanifold of $\Sigma$, and such that $d G$ equals $\beta^{\prime \prime}$ in the directions tangent to $r D^{n-k}$ at every point of $D^{k}$. Taking $M^{\prime}=G\left(D^{k} \times r D^{n-k}\right)$ and $K^{\prime}=\bar{W} \cup M^{\prime}$ reduces the proof to the case $k=n$. (The fact that $\partial M^{\prime}$ is not entirely contained in $\partial W$ does not cause any complication.)

\section{THE CASE $\operatorname{dim}_{\mathbb{R}} X \neq 4$}

In this section we prove our main results in the case when $\operatorname{dim}_{\mathbb{R}} X \neq 4$. Let $P$ be a compact Hausdorff space and let $X$ and $Y$ be smooth manifolds. A $P$-map from $X$ to $Y$ is a continuous map $f: X \times P \rightarrow Y$. If $X$ and $Y$ are complex manifolds then such $f$ is said to be a holomorphic $P$-map if $f_{p}=f(\cdot, p): X \rightarrow Y$ is holomorphic for every fixed $p \in P$.

Theorem 6.1. Let $(X, J)$ be a smooth almost complex manifold of real dimension $2 n$ which is exhausted by a Morse function $\rho: X \rightarrow \mathbb{R}$ without critical points of index $>n$. Assume that for some $c \in \mathbb{R}$ the structure $J$ is integrable in $X_{c}=\{x \in X: \rho(x)<c\}$ and $\rho$ is strongly J-plurisubharmonic in $X_{c}$. Let $Y$ be a complex manifold with a distance function $d_{Y}$ induced by a Riemannian metric. Let $P$ be a compact Hausdorff space and $f: X \times P \rightarrow Y$ be a P-map which is J-holomorphic in $X_{c}$.

If $n \neq 2$, or if $n=2$ and $\rho$ has no critical points of index $>1$ in $\{x \in$ $X: \rho(x) \geq c\}$ then for every compact set $K \subset X_{c}$ and for every $\epsilon>0$ there exist a Stein structure $\widetilde{J}$ on $X$ and a homotopy of $P$-maps $f^{t}: X \times P \rightarrow Y$ $(t \in[0,1])$ satisfying the following properties:

(a) $f^{0}=f$.

(b) The P-map $\tilde{f}:=f^{1}$ is $\widetilde{J}$-holomorphic on $X$. 
(c) There is a homotopy $J_{t}$ of almost complex structures on $X$ which is fixed in a neighborhood of $K$ such that $J_{0}=J$ and $J_{1}=\widetilde{J}$; if $J$ is integrable on $X$ then $J_{t}$ can be chosen integrable for all $t \in[0,1]$.

(d) For every $t \in[0,1]$ the $P$-map $f^{t}$ is $J$-holomorphic in a neighborhood of $K$ and satisfies $\sup \left\{d_{Y}\left(f^{t}(x, p), f(x, p)\right): x \in K, p \in P\right\}<\epsilon$.

The special case of Theorem 6.1, when applied to the constant map $X \rightarrow$ point, coincides with Theorem 1.3.1 of Eliashberg [7].

Proof. We shall present the proof for the nonparametric case since the parameters do not present any essential complication.

Fix a compact set $K \subset X_{c}$ and choose a regular value $c_{0} \in \mathbb{R}$ of $\rho$ such that $K \subset X_{c_{0}} \Subset X_{c}$. Hence the structure $J=J_{0}$ is integrable in a neighborhood of $\bar{X}_{c_{0}}$ and the map $f: X \rightarrow Y$ is $J_{0}$-holomorphic in a neighborhood of $\bar{X}_{0}$.

Let $p_{1}, p_{2}, \ldots$ be the critical points of $\rho$ in $\left\{x \in X: \rho(x)>c_{0}\right\}$, ordered so that $\rho\left(p_{j}\right)<\rho\left(p_{j+1}\right)$ for every $j$. Choose numbers $c_{j}$ satisfying $c_{-1}=$ $-\infty<c_{0}<\rho\left(p_{1}\right)<c_{1}<\rho\left(p_{2}\right)<c_{2}<\ldots$. Let $k_{j}$ denote the Morse index of $p_{j}$, so $k_{j} \leq n$. For each $j=0,1, \ldots$ we set $X_{j}=\left\{x \in X: \rho(x)<c_{j}\right\}$, $\Sigma_{j}=\partial X_{j}=\left\{x: \rho(x)=c_{j}\right\}$, and $A_{j}=\left\{x \in X: c_{j-1} \leq \rho(x)<c_{j}\right\}$. We shall inductively construct a sequence of almost complex structures $J_{j}$ on $X$ and a sequence of maps $f_{j}: X \rightarrow Y$ satisfying the following for $j=0,1,2, \ldots$ :

(i) $J_{j}$ is integrable in a neighborhood of $\bar{X}_{j}$ and the manifold $\left(X_{j}, J_{j}\right)$ is Stein with strongly pseudoconvex boundary,

(ii) $J_{j}=J_{j-1}$ in a neighborhood of $\bar{X}_{j-1}$,

(iii) the set $\bar{X}_{j-1}$ is $\mathcal{H}\left(X_{j}, J_{j}\right)$-convex,

(iv) the map $f_{j}$ is $J_{j}$-holomorphic in a neighborhood of $\bar{X}_{j}$,

(v) $\sup _{x \in X_{j-1}} d\left(f_{j}(x), f_{j-1}(x)\right)<\epsilon 2^{-j-1}$, and

(vi) there is a homotopy from $f_{j-1}$ to $f_{j}$ which is $J_{j}$-holomorphic and uniformly close to $f_{j-1}$ in a neighborhood of $\bar{X}_{j-1}$ (satisfying the estimate in (v)).

These conditions clearly hold for $j=0$, and in this case (ii), (iii) and (v) are vacuous. Assume inductively that the above hold for $j-1$. By Morse theory $X_{j}$ is diffeomorphic to a handlebody obtained by attaching to $X_{j-1}$ an embedded disc $M_{j} \subset X \backslash X_{j-1}$ of dimension $k_{j}$ and smoothly thickening the union $\bar{X}_{j-1} \cup M_{j}$ inside $X_{j}$. ( $M_{j}$ may be taken as the unstable manifold of the critical point $p_{j}$ for the gradient flow of $\rho$.)

Applying Lemmas 3.1 and 5.1 with $W=X_{j-1}, J=J_{j-1}$ and $f=f_{j-1}$ we get a Stein structure $\widetilde{J}$ on a handlebody $\widetilde{W}$ which is isotopic to $X_{j}$ and satisfies $\bar{X}_{j-1} \subset \widetilde{W} \subset X_{j}$, and a map $\tilde{f}: X \rightarrow Y$, homotopic to $f_{j-1}$, which is $\widetilde{J}$-holomorphic on $\widetilde{W}$ and which approximates $f_{j-1}$ uniformly on $X_{j-1}$. (If $k_{j}=0$, a new connected component of the sublevel set $\{\rho<c\}$ appears at $p_{j}$ when $c$ passes the value $\rho\left(p_{j}\right)$, and it is trivial to find $\widetilde{f}$ and $\widetilde{J}$ with these properties.) There is a smooth diffeotopy $h_{t}: X \rightarrow X(t \in[0,1])$ which is 
fixed in a neighborhood of $\bar{X}_{j-1}$ such that $h_{0}$ is the identity map on $X$ and $h=h_{1}$ satisfies $h\left(X_{j}\right)=\widetilde{W}$. Taking $J_{j}=h^{*}(\widetilde{J})$ and $f_{j}=\widetilde{f} \circ h$ completes the inductive step. (The homotopy from $f_{j-1}$ to $f_{j}$ is obtained by composing the homotopy from $f_{j-1}$ to $\tilde{f}$ by the map $h$.) The induction may proceed.

By properties (i) and (ii) there is a unique integrable complex structure $\widetilde{J}$ on $X$ which agrees with $J_{j}$ on $X_{j}$. By the construction $\widetilde{J}$ is homotopic to the initial structure $J=J_{0}$ since at the $j$-th stage of the construction, the structure $J_{j}$ was chosen homotopic to $J_{j-1}$ by a homotopy which is fixed on a neighborhood of $\bar{X}_{j-1}$. The complex manifold $(X, \widetilde{J})$ is exhausted by the increasing sequence of Stein domain $X_{j}$, and the Runge property (iii) implies that $(X, \widetilde{J})$ is Stein. Properties (iv) and (v) insure that the sequence $f_{j}: X \rightarrow Y$ converges uniformly on compacts in $X$ to the $\widetilde{J}$-holomorphic map $\widetilde{f}=\lim _{j \rightarrow \infty} f_{j}: X \rightarrow Y$ satisfying $\sup _{x \in X_{0}} d\left(\widetilde{f}(x), f_{0}(x)\right)<\epsilon$. By (vi) the homotopies from $f_{j-1}$ to $f_{j}$ also converge, uniformly on compacts in $X$, and give a homotopy from the initial map $f_{0}$ to $\tilde{f}$, thus completing the proof.

If the initial structure $J$ on $X$ is integrable then all steps can be made within the class of integrable structures.

Proof of Theorem 1.2. This requires only minor modifications of the proof of Theorem 6.1. The main difference is that we do not change the given integrable structure $J$ during the construction, at the cost of remaining on subsets of $X$ which are only diffeomorphic to sublevel sets of $\rho$ (and not equal to them as before). We will in fact obtain a stronger version with approximation, similar to Theorem 6.1.

We assume the same assumptions and notation as in the proof of Theorem 6.1. Thus, $W_{0}=X_{0}$ is a sublevel set of a Morse exhaustion function $\rho: X \rightarrow \mathbb{R}$ which has no critical points of index $>n$ in $X \backslash W_{0}, \rho$ is strongly plurisubharmonic in a neighborhood of $\bar{W}_{0}$, and the initial map $f_{0}=f: X \rightarrow Y$ is holomorphic in a neighborbood of $\bar{W}_{0}$. Let $X_{j}=\left\{\rho<c_{j}\right\}$ where the constants $c_{j}$ are chosen as in the proof of Theorem 6.1, so $\rho$ has a unique critical point $p_{j}$ in $X_{j} \backslash X_{j-1}$. Choose $\epsilon>0$ and let $d_{Y}$ denote a distance function on the manifold $Y$. Assuming that $n \neq 2$ (the case $n=2$ will be treated in $\S 7$ below) we inductively construct an increasing sequence of relatively compact, strongly pseudoconvex domains $W_{1} \subset W_{2} \subset \cdots \subset X$ with smooth boundaries, a sequence of maps $f_{j}: X \rightarrow Y$, and a sequence of diffeomorphisms $h_{j}: X \rightarrow X$ such that the following hold for all $j=1,2, \ldots$ :

(i) $\bar{W}_{j-1}$ is $\mathcal{H}\left(W_{j}\right)$-convex,

(ii) $f_{j}$ is holomorphic in a neighborhood of $\bar{W}_{j}$ and is homotopic to $f_{j-1}$ by a homotopy $f_{j, t}: X \rightarrow Y(t \in[0,1])$ such that each $f_{j, t}$ is holomorphic near $\bar{W}_{j-1}$ and satisfies $\sup _{x \in W_{j-1}} d_{Y}\left(f_{j, t}(x), f_{j-1}(x)\right)<\epsilon 2^{-j}$,

(iii) $h_{j}\left(X_{j}\right)=W_{j}$, and 
(iv) $h_{j}=g_{j} \circ h_{j-1}$ where $g_{j}: X \rightarrow X$ is a diffeomorphism of $X$ which is diffeotopic to $i d_{X}$ by a diffeotopy which is fixed in a neighborhood of $\bar{W}_{j-1}$. (In particular, $h_{j}$ agrees with $h_{j-1}$ near $\bar{W}_{j-1}$.)

Granted such sequences, it is easily verified that the limit map

$$
\tilde{f}=\lim _{j \rightarrow \infty} f_{j}: \Omega=\cup_{j=1}^{\infty} W_{j} \rightarrow Y
$$

and the limit diffeomorphism $h=\lim _{j \rightarrow \infty} h_{j}: X \rightarrow \Omega$ satisfy the conclusion of Theorem 1.2 .

To prove the inductive step we begin by attaching to $W_{j-1}=h_{j-1}\left(X_{j-1}\right)$ the disc $M_{j}:=h_{j-1}\left(D_{j}\right)$, where $D_{j} \subset X_{j} \backslash X_{j-1}$ (with $\partial D_{j} \subset \partial X_{j-1}$ ) is the unstable disc for $\rho$ at the unique critical point $p_{j} \subset X_{j} \backslash X_{j-1}$ of $\rho$ in this region. By Lemma 3.1 we can isotope $M_{j}$ to a totally real, real analytic disc in $X$ attached to $\partial W_{j-1}$ along a Legendrian sphere. Applying Lemma 5.1 with the integrable structure $J$ we find the next map $f_{j}: X \rightarrow Y$ which is holomorphic in a thin handlebody $W_{j} \supset \bar{W}_{j-1} \cup M_{j}$. The next diffeomorphism $h_{j}$ with the stated properties is then furnished by the Morse theory. This concludes the proof of Theorem 1.2 .

With a bit more care one can insure that $\partial \Omega$ is smoothly bounded and strongly pseudoconvex, but in general we cannot choose such $\Omega$ to be relatively compact, unless $X$ admits an exhaustion function $\rho: X \rightarrow \mathbb{R}$ with at most finitely many critical points.

In the remainder of this section we discuss the existence of holomorphic maps of maximal rank (immersions resp. submersions). Let $X$ and $Y$ be complex manifolds. A necessary condition for a continuous map $f: X \rightarrow Y$ to be homotopic to a holomorphic map of maximal rank is that $f$ is covered by a complex vector bundle map $\iota: T X \rightarrow T Y$, i.e., such that for every $x \in X$ the map $\iota_{x}: T_{x} X \rightarrow T_{f(x)} Y$ is $\mathbb{C}$-linear and of maximal rank. If $X$ is Stein, this condition is known to be also sufficient in the following cases:

(i) $\operatorname{dim} X=1$ and $Y=\mathbb{C}$ (Gunning and Narasimhan 38]);

(ii) $Y=\mathbb{C}^{q}$ with $q>\operatorname{dim} X$ (Eliashberg and Gromov [9, 36]);

(iii) $Y=\mathbb{C}^{q}$ with $q<\operatorname{dim} X$ (Forstnerič [17]);

(iv) $n=\operatorname{dim} X \geq \operatorname{dim} Y$ and $Y$ satisfies a Runge approximation property for holomorphic submersion $\mathbb{C}^{n} \rightarrow Y$ on compact convex sets in $\mathbb{C}^{n}$ (the Property $S_{n}$ in [18]).

By an obvious modification of the proof of Theorem 6.1 using the part of Lemma 5.1 for maps of maximal rank, one obtains the following result which in particular implies Theorem 1.4. We leave out the details.

Theorem 6.2. Let $(X, J)$ be a smooth almost complex manifold of real dimension $2 n$, exhausted by a Morse function $\rho: X \rightarrow \mathbb{R}$ without critical points of index $>n$. Let $f: X \rightarrow Y$ be a continuous map to a complex manifold $Y$, and let $\iota: T X \rightarrow T Y$ be a complex vector bundle map covering $f$ such that $\iota_{x}: T_{x} X \rightarrow T_{f(x)} Y$ is of maximal rank $\min \{\operatorname{dim} X, \operatorname{dim} Y\}$ for 
every $x \in X$. If $n \neq 2$, or if $n=2$ and $\rho$ has no critical points of index $>1$, there is a homotopy $\left(J_{t}, f_{t}, \iota_{t}\right)(t \in[0,1])$ where $J_{t}$ is an almost complex structure on $X, f_{t}: X \rightarrow Y$ is a continuous map, and $\iota_{t}: T X \rightarrow T Y$ is a $J_{t}$-complex linear vector bundle map of pointwise maximal rank covering $f_{t}$, such that the following hold:

(i) $J_{0}=J, f_{0}=f, \iota \iota_{0}=\iota$,

(ii) $\left(X, J_{1}\right)$ is a Stein manifold,

(iii) the map $f_{1}: X \rightarrow Y$ is $J_{1}$-holomorphic and of maximal rank (an immersion resp. a submersion), and $d f_{1}=\iota_{1}$.

If in addition there is a constant $c \in \mathbb{R}$ such that $J$ is integrable Stein on the set $X_{c}=\{\rho<c\}, f$ is holomorphic on $X_{c}$ and $\iota=d f$ on $X_{c}$ then for every compact set $K \subset X_{c}$ the homotopy $J_{t}$ may be chosen fixed near $K$, the map $f_{t}$ may be chosen holomorphic near $K$ and uniformly close to $f=f_{0}$ on $K$, and $\iota_{t}$ may be chosen to satisfy $\iota_{t}=d f_{t}$ near $K$ for each $t$.

The analogous result holds for a family of maps parametrized by a compact Hausdorff space (compare with Theorem 6.1).

\section{The FOUR DIMENSIONAL CASE}

The following is a precise version of Theorem 1.1 in the case $\operatorname{dim}_{\mathbb{R}} X=4$. The notion of a $P$-map was defined at the beginning of $\S 6$.

Theorem 7.1. Let $X$ be a smooth oriented 4-manifold, exhausted by a Morse function $\rho: X \rightarrow \mathbb{R}$ without critical points of index $>2$. Assume that for some $c \in \mathbb{R}$ there is an integrable complex structure $J$ on $X_{c}=$ $\{x \in X: \rho(x)<c\}$ such that $\left.\rho\right|_{X_{c}}$ is strongly J-plurisubharmonic. Let $Y$ be a complex manifold with a distance function $d_{Y}$ induced by a Riemannian metric, let $P$ be a compact Hausdorff space, and let $f: X \times P \rightarrow Y$ be a $P$-map which is J-holomorphic in $X_{c}$.

Given a compact set $K \subset X_{c}$ and an $\epsilon>0$, there are a Stein surface $\left(X^{\prime}, J^{\prime}\right)$, an orientation preserving homeomorphism $h: X \rightarrow X^{\prime}$ which is biholomorphic in a neighborhood of $K$, and a holomorphic $P$-map $f^{\prime}: X^{\prime} \times$ $P \rightarrow Y$ such that the $P$-map $\widetilde{f}: X \times P \rightarrow Y$, defined by $\widetilde{f}(x, p)=f^{\prime}(h(x), p)$, is homotopic to $f$ and satisfies

$$
\sup \left\{d_{Y}(f(x, p), \widetilde{f}(x, p)): x \in K, p \in P\right\}<\epsilon .
$$

Unlike in the case $n>2$, we do not need to assume that the almost complex structure $J$ is defined on all of $X$ since the obstruction to extending $J$ across an attached handle only appears for handles of index $>2$. However, if $J$ is already given on all of $X$, one can choose $\left(X^{\prime}, J^{\prime}\right)$ such that the homotopy class of almost complex structures on $X$ determined by $h^{*}\left(J^{\prime}\right)$ equals the class of $J$; Gompf showed that this notion makes sense under orientation preserving homeomorphisms [27, p. 645]. 
Before proving Theorem 7.1 we indicate some consequences. The following is obtained by combining Theorem 7.1 with Corollary 3.2 and Theorem 3.3 of Gompf [27, p. 648].

Corollary 7.2. Let $X$ be a smooth, closed, oriented 4-manifold. There exists a smooth, finite wedge of circles $\Gamma \subset X$ such that for every continuous map $f: X \backslash \Gamma \rightarrow Y$ to a complex manifold $Y$ there is a (possibly exotic) Stein structure on $X \backslash \Gamma$ and a holomorphic map $\tilde{f}: X \backslash \Gamma \rightarrow Y$ homotopic to $f$. If $X=\mathbb{C P}^{2}$, this holds after removing a single point (in this case any Stein structure on $\mathbb{C P}^{2} \backslash\{p\}$ is exotic). The analogous result holds for each open oriented 4-manifold after removing a suitably chosen smooth 1-complex.

The point is that there is a wedge of circles $\Gamma$ in $X$ such that $X \backslash \Gamma$ admits a handle decomposition without 3 - and 4 -handles. The projective plane $\mathbb{C P}^{2}$ has a single 4-cell (and no 3-cells) in its handlebody decomposition, hence removing a point leaves only cells of index $\leq 2$.

Here is another result obtained by combining Gompf's methods in [28] with the proof of Theorem 1.2 (ii) given below.

Corollary 7.3. Let $M$ be a tame, topologically embedded $C W$ 2-complex in a complex surface $X$ and let $U$ be an open neighborhood of $M$ in $X$. For every continuous map $f: M \rightarrow Y$ to a complex manifold $Y$ there exist a topological isotopy $h_{t}: X \rightarrow X$, with $h_{0}=i d_{X}$ and $h_{t}(M) \subset U$ for all $t \in[0,1]$, a Stein thickening $\Omega \subset U$ of the $C W$ complex $h_{1}(M)$, and a holomorphic map $\tilde{f}: \Omega \rightarrow Y$ such that $\tilde{f} \circ h_{1}: M \rightarrow Y$ is homotopic to $f$.

Gompf showed that the necessary adjustment of the initial 2-complex $M$ in $X$ is quite mild from the topological point of view, and all essential data of the topological embedding $M \hookrightarrow X$ can be preserved. Stein domains $\Omega$ obtained in this way will typically have nonsmooth boundaries in $X$ and may be chosen to realize uncountably many distinct diffeomorphism types. In certain special cases when the 2-cells in $M$ satisfy certain framing conditions, it is possible to find Stein thickenings of a $\mathcal{C}^{0}$-small smooth perturbation of $M$ in $X$ which even have the diffeomorphism type of a smooth handlebody with core $M$. In this direction see also Costantino [5].

Proof of Theorem 7.1. We shall follow Gompf's construction of exotic Stein structures [27], but with a modification which will better suit our task of finding a holomorphic map in the given homotopy class. Subsequently we will show how the construction can be carried out inside a given complex surface as in [28], thereby proving Theorem 1.2 (for $\left.\operatorname{dim}_{\mathbb{R}} X=4\right)$ and Corollary 7.3 . 
The proof of Theorem 6.1 applies without any changes when attaching handles of index zero or one, but the difficulty arises when attaching 2handles because Lemma 3.1 may fail to give an embedded core 2-disc attached along a Legendrian curve. As shown by Gompf [27, 28] the obstruction can be removed by using Casson handles, but the price to pay is a change of the underlying smooth structure on $X$.

We begin by reviewing the necessary background material, referring to [27] for a more complete discussion. Let $W$ be a relatively compact, smoothly bounded domain in $X$ such that $J$ is defined on a neighborhood of $\bar{W}$ and $\Sigma=\partial W$ is strongly $J$-pseudoconvex. Let $G: D=D^{2} \hookrightarrow X \backslash W$ be an embedded 2-disc attached along the circle $G(S) \subset \Sigma:=\partial W$. Let $g=$ $\left.G\right|_{S}: S \hookrightarrow \Sigma$. The restriction of the contact subbundle $\xi=T \Sigma \cap J(T \Sigma)$ to the circle $C:=g(S)$ is a trivial bundle (every oriented two-plane bundle over a circle is trivial). As in $\S 3$ above we can use the Legendrization theorem to make $G$ normal to $\partial W$ and its boundary circle $C \subset \Sigma$ Legendrian in $\Sigma$. (The boundary circle remains in $\Sigma$ during this isotopy.) We denote by $M=G(D)$ the resulting embedded 2-disc in $X \backslash W$, with $\partial M=C$.

Let $\left.\nu_{C} \subset T \Sigma\right|_{C}$ denote the normal bundle of $C$ in $\Sigma$. It is spanned by the pair of vector fields $(J w, J \tau)$ where $w$ is normal to $\Sigma$ in $X$, with $J w \in T \Sigma$, and $\tau$ is tangent to $C$. The pair $(J w, J \tau)$ is a canonical framing, or a Thurston-Bennequin framing, $T B$, of the normal bundle $\nu_{C}$. (This notion is only defined for Legendrian knots or links.)

Let $\beta:\left.\nu \rightarrow T X\right|_{M}$ denote a normal framing over $M$ (a trivialization of the normal bundle of $M$ in $X)$, chosen such that $\beta\left(\left.\nu\right|_{S}\right)=\nu_{C}$. We thus have two framings of $\nu_{C}$, namely $\beta$ (which extends to the disc $M$ ) and the $T B$ framing. Since $\nu_{C}$ is a trivial 2-plane bundle over $C$, any two framings differ up to homotoy by a map $C \rightarrow S O(2)=S^{1}$, hence by an integer. We can thus write $[\beta]=T B+k$; the integer $k=k([\beta]) \in \mathbb{Z}$ will be called the framing index of $\beta$.

In the model case when $M=D^{2} \subset \mathbb{C}^{2}$ is the core of a standard handle in $\mathbb{C}^{2}$ attached to a quadric domain $Q_{\lambda} \subset \mathbb{C}^{2}(2.2)$ we easily see that

$$
[\beta]=T B-1 \text {. }
$$

Indeed, the tangent field $\tau$ to $S=\partial D^{2}$ rotates once in the positive (counterclockwise) direction as we trace $S$ in the positive direction. Since the complex structure operator $J_{s t}$ on $T_{z} \mathbb{C}^{2}$ is an orientation reversing map of $\mathbb{R}^{2}$ onto $i \mathbb{R}^{2}$, the vector field $J \tau$ (which determines $T B$ ) rotates once in the clockwise direction, hence $\beta$ is obtained from the $T B$ framing by one left (negative) twist, so (7.1) holds.

When the normal framing $\beta$ of $M$ satisfies (7.1) then $J$ extends to an integrable complex structure in a neighborhood of $\bar{W} \cup M$ in $X$ such that the core $\operatorname{disc} M$ is $J$-real (this is precisely as in [7]). In this case Lemma 5.1 in $\S 5$ applies and yields a holomorphic map in a neighborhood of $\bar{W} \cup M$ which approximates the previous map uniformly on $W$. If this ideal situation 
occurs for all 2-handles in $X \backslash W$ then the construction of a Stein structure on $X$, and of a holomorphic map $X \rightarrow Y$ in a given homotopy class, can be completed exactly as in $\S 6$.

Suppose now that $k=[\beta]-T B \neq-1$ for some 2-handle. A basic fact from the theory of Legendrian knots [2, 8] is that for any Legendrian knot $K$ there is a $\mathcal{C}^{0}$-small isotopy preserving the knot type, but changing its Legendrian knot type, which adds a desired number of left (negative) twists to the $T B$ framing. (One adds small spirals to $K$.) Since the homotopy class of the $\beta$ framing is preserved under an isotopy of $C$ in $\Sigma$, we see that $k=[\beta]-T B$ can be increased by any number of units. If $k<-1$, it is therefore possible to add spirals to the boundary circle and obtain an isotopic embedding $(D, S) \hookrightarrow(X \backslash W, \Sigma)$ satisfying (7.1), thereby reducing the problem to the previous case.

The problem is more difficult when $k \geq 0$ since it is in general impossible to add right twists to the $T B$ framing (equivalently, to decrease the framing index $k$ ). This is only possible in a contact structure which is overtwisted, in the sense that it contains a topologically unknotted Legendrian knot $K$ with the Thurston-Bennequin index $t b(K)=0$; adding such knot to a Legendrian knot adds a positive twist to the $T B$ framing, making it possible to decrease $k=[\beta]-T B$ and hence reach $k=-1$. However, Eliashberg proved in [8] that contact structures arising as boundaries of strongly pseudoconvex Stein manifolds are never overtwisted (they are tight). A 2-handle for which we cannot find an isotopy of the boundary circle to a Legendrian knot so that (7.1) holds will be called in the sequel a wrongly attached handle.

In [27] Gompf showed how one can circumvent the problem by replacing each wrongly attached 2-handle by a Casson handle which is homeomorphic, but not diffeomorphic, to the standard 2-handle $D^{2} \times D^{2}$. In such case the new domain can be chosen to admit a Stein structure and is homeomorphic, but not diffeomorphic, to the original manifold. In the following two paragraphs we summarize Gompf's construction for future reference.

Let $Z$ be a smooth 4-manifold obtained by attaching a 2-handle $h$, with the core $\operatorname{disc} M$, to a compact Stein surface $\bar{W}$ along its strongly pseudoconvex boundary $\Sigma=\partial W$. We first isotope $C=\partial M \subset \Sigma$ inside $\Sigma$ to a Legendrian knot. Since the $T B$ invariant can be increased by a nonLegendrian isotopy by an arbitrary integer, while the homotopy class $[\beta]$ does not change by the isotopy, we can assume that the framing coefficient $[\beta]-T B$ is odd, $[\beta]-T B=-1+2 k$ for some $k \in \mathbb{Z}$. If $k<0$, we can isotope the boundary of $h$ (by adding left twists to the $T B$ framing) to get (7.1) and we are done. If not, we remove the 2-handle $h$ and reattach it to $W$ along $C$ using the framing $T B-1$, meaning that we add $2 k$ negative twists to the framing of $h$. Lets us call this new handle $h^{\prime}$, and let $Z^{\prime}$ be the new manifold obtained by attaching $h^{\prime}$ to $\bar{W}$ in this way. As mentioned above, we can extend the Stein structure from $\bar{W}$ across the 2-handle $h^{\prime}$ to a Stein structure on $Z^{\prime}$. The problem is that the manifold $Z^{\prime}$ is not diffeomorphic 
to $Z$. In fact, if the 2-handle $h$ in the manifold $Z$ gives a homology class in $H_{2}(Z, \mathbb{Z})$ with self-intersection $m$, the self-intersection of the handle $h^{\prime}$ in the homology of $Z^{\prime}$ equals $m-2 k$. In order to compensate this error we now make $k$ positive self-plumbings to the handle $h^{\prime} \subset Z^{\prime}$ to get a yet new manifold $Z_{1}$. (A positive self-plumbing is done by choosing two disjoint closed discs $\triangle_{1}, \triangle_{2}$ in the core disc $M$ of the handle $h^{\prime}$, trivializing the normal bundle of $M$ over these discs to get subsets $\triangle_{1} \times \triangle_{1}^{\prime}, \triangle_{2} \times \triangle_{2}^{\prime}$ of $Z^{\prime}$ diffeomorphic to the standard handle $D^{2} \times D^{2}$, and identifying them by the map $\triangle_{1} \times \triangle_{1}^{\prime} \rightarrow \triangle_{2} \times \triangle_{2}^{\prime}$ that interchanges the factors: $(z, w) \mapsto(w, z)$ in the coordinates on $D^{2} \times D^{2}$. (For a negative plumbing we would use $(z, w) \rightarrow(\bar{w}, z)$.) The manifold $Z_{1}$ is the quotient space of $Z^{\prime}$ under the above identification. The image of $M$ is an immersed 2-disc $M_{1}$ in $Z_{1}$ with exactly one positive transverse double point $p$ (the image of the centers $p_{1}$ resp. $p_{2}$ of the discs $\triangle_{1}$ resp. $\triangle_{2}$ ). The self-plumbing can be done so that in local coordinates near $p, M_{1}$ equals $\mathbb{R}^{2} \cup i \mathbb{R}^{2} \subset \mathbb{C}^{2}$, with $p$ corresponding to the origin, and $Z_{1}$ is a tubular neighborhood of this set. Since $\mathbb{R}^{2} \cup i \mathbb{R}^{2}$ admits a basis of tubular Stein neighborhoods in $\mathbb{C}^{2}$ (see [7, Theorem 1.3.5.] or [16. Theorem 2.2]), we get a Stein structure on $Z_{1}$ which agrees with the original structure inherited from $Z^{\prime}$ away from the plumbed double point $p$. Although $Z_{1}$ is not even homotopically equivalent to $Z$, we do have $H_{2}(Z, \mathbb{Z})$ isomorphic to $H_{2}\left(Z_{1}, \mathbb{Z}\right)$ by an isomorphism preserving the self-intersection form. This follows from the fact that a positive self-plumbing of a handle $h^{\prime}$ introduces a positive transverse double point to its core, thus raising the self-intersection number of $h^{\prime}$ by 2 .

In the next step we show that $Z$ can be reconstructed back from the manifold $Z_{1}$ by attaching additional 2-cells to $Z_{1}$. The group $H_{1}\left(Z_{1}, \mathbb{Z}\right)$ differs from $H_{1}(Z, \mathbb{Z})$ by $\mathbb{Z}^{k}$, with new homology classes represented by one loop in $Z_{1}$ for each performed plumbing, the only requirement being that the loop passes once through the plumbed double point. Attaching a 2-handle to $Z_{1}$ along each such loop cancels the extra homology and moreover, if the framing is correct, reconstructs the original manifold $Z$. The details can be found in [27, but will also be obvious from the construction below. The problem now is that the framing of the 2-handles which we need to attach to $Z_{1}$ (in order to reconstruct $Z$ ) may not be correct, in the sense that the attaching circles of the core discs cannot be isotoped to Legendrian knots satisfying (7.1). To correct this, one repeats the above steps, adding kinks to each of these wrongly attached handles, thus beginning the Casson tower procedure. In this way one gets an increasing sequence of Stein manifolds $X_{1} \subset X_{2} \subset X_{3} \subset \cdots$ with strongly pseudoconvex boundaries, each of them Runge in the next one. The limit manifold $\cup_{j} X_{j}$ is then also Stein, and by Freedman's result on Casson handles [25] it is homeomorphic to the original manifold $X$. 
Here is a somewhat different explanation of the above procedure which is better suited to our purpose; its main advantage is that we remain inside the same manifold $X$ during the entire construction.

Let us take an immersed totally real sphere $S^{2} \rightarrow \mathbb{C}^{2}$ with a positive double point at $0 \in \mathbb{C}^{2}$ and no other double points; an explicit Lagrangian example is due to Weinstein [57]:

$$
F(x, y, z)=(x(1+2 i z), y(1+2 i z)) \in \mathbb{C}^{2}
$$

where $(x, y, z) \in \mathbb{R}^{3}, x^{2}+y^{2}+z^{2}=1$. Let us think of $S^{2}$ as the union of two closed discs $D_{0} \cup D_{\infty}$, glued along their boundary circles $S^{1}=D_{0} \cap D_{\infty}$ and chosen such that $F\left(D_{\infty}\right) \subset \mathbb{C}^{2}$ is embedded while $F\left(D_{0}\right)$ contains the positive double point at the origin. The oriented normal bundle $\nu$ of $F$ is isomorphic to $\overline{T S^{2}}$, the tangent bundle of $S^{2}$ with the reversed orientation. Indeed, $\left.T \mathbb{C}^{2}\right|_{F\left(S^{2}\right)}=F_{*}\left(T S^{2}\right) \oplus \nu$, and the complex structure $J_{s t}$ gives an orientation reversing isomorphism of the first onto the second summand. By a small modification of $F$ we may assume that the double point at 0 (the center of the kink) is special (4.1), meaning that in a suitable local holomorphic coordinate system the image equals $\mathbb{R}^{2} \cup i \mathbb{R}^{2}$ near the origin. We shall take $K=F\left(D_{0}\right) \subset \mathbb{C}^{2}$ as our standard kink which will be used to correct the framing coefficient of wrongly attached handles.

Since $T S$ has the Euler number $\chi(T S)=2$, the normal bundle $\nu$ of the Weinstein sphere in $\mathbb{C}^{2}$ has $\chi(\nu)=-2$; hence a copy $K$, glued into a 2-disc $M$ attached to $\partial W$ along a Legendian knot as in Fig. 8, will reduce the framing coefficient of $M$ by two units. This is seen explicitly as follows. Thinking of $S^{2}$ as the Riemann sphere $\mathbb{C} \cup\{\infty\}$, with $D_{0}=\{z \in \mathbb{C}:|z| \leq 1\}$ and $D_{\infty}=\{w \in \mathbb{C} \cup\{\infty\}:|w| \geq 1\}$, the real and the imaginary part of the complex vector field $\frac{\partial}{\partial w}$ provide a reference framing for $\left.T S\right|_{D_{\infty}}$. From $\frac{\partial}{\partial w}=-z^{2} \frac{\partial}{\partial z}$ we see that $\frac{\partial}{\partial w}$ makes two left (negative) twists when compared to the framing $\frac{\partial}{\partial z}$ for $\left.T S^{2}\right|_{D_{0}}$ as we trace the circle $S=\partial D_{0}=\{|z|=1\}$ in the positive direction. Conversely, $\frac{\partial}{\partial z}$ makes two right (positive) twists in comparison to $\frac{\partial}{\partial w}$. Considering these framings on the immersed sphere $F\left(S^{2}\right) \subset \mathbb{C}^{2}$ and applying $J_{s t}$ we obtain two framings of the normal bundle $\nu$ over the respective discs. Due to the reversal of the orientation under $J_{s t}$ we see that the framing for $\left.\nu\right|_{D_{0}}$ makes two left (negative) twists when compared to the framing for $\left.\nu\right|_{D_{\infty}}$, which explains $\chi(\nu)=-2$.

With $F$ as in (7.2), let $\Delta=\left\{F(0, y, z): y \geq 0, y^{2}+z^{2} \leq 1\right\} \subset \mathbb{C}^{2}$. This 2-disc is embedded in $\mathbb{C}^{2}$, except along the side $\{y=0\}$ which gets pinched to $0 \in \mathbb{C}^{2}$. Note that $\partial \Delta \subset F\left(S^{2}\right)$, and the union $F\left(S^{2}\right) \cup \Delta$ has a tubular neighborhood diffeomorphic to $S^{2} \times \mathbb{R}^{2}$.

In order to make a self-intersection at a point $p$ in the core disc $M$ of a handle $h$ in our 4-manifold $X$, we replace a small disc in $M$ around $p$ by a copy of the standard kink $K$. (See Fig. 8, we removed the small dotted disc and smoothly attached along its boundary the kinky disc shown 
on the right.) We have seen that this surgery reduces the relative Euler number over the immersed disc $M$ by 2 for each kink. Adding $k$ kinks on $M$ inside $X$ and then taking a tubular neighborhood has the same effect as first removing the handle $h$ from $X$, reattaching it with a framing of the boundary reduced by $2 k$, and then performing $k$ self-plumbings on $h$ (as was done by Gompf [27] and described above). In this way we see that the manifold $Z_{1}$, constructed above when discussing Gompf's proof, can be seen as a submanifold of the original manifold $X$, changed only by a surgery in a small coordinate neighborhood of each of the kinked points on the core disc of the handle $h$. We can also explicitly see the trivializing 2-cell $\Delta$ that needs to be added to each of the kinks in order to reconstruct the desired manifold.

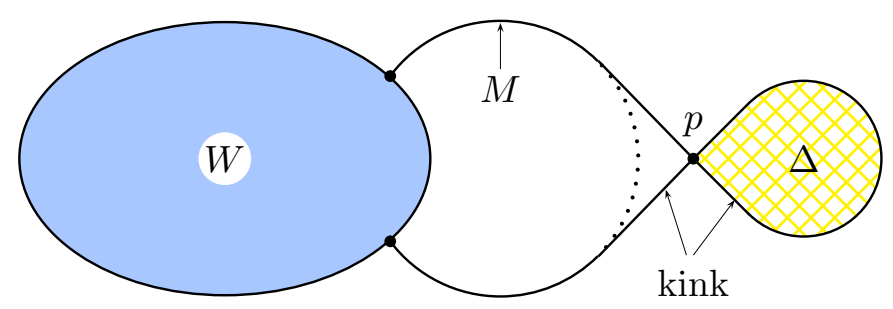

Figure 8. A kinky disc $M$ with a trivializing 2-cell $\Delta$

In the next stage of the construction every such disc $\Delta$ will also have to receive a kink in order to correct its framing coefficient. This begins the Casson tower procedure which will converge to a Casson handle in place of the original removed disc in $M$.

We are now ready to complete the proof of Theorem 7.1. Assume that our 4-manifold $X$ is constructed by successively attaching handles $h_{1}, h_{2}, h_{3}, \ldots$ of index $\leq 2$, beginning with the compact domain $\bar{W} \subset X$ with smooth boundary $\Sigma=\partial W$. By assumption we also have an integrable complex structure $J$ in a neighborhood of $\bar{W}$ such that $W$ is Stein and its boundary $\Sigma$ is strongly pseudoconvex. Let $M_{1}, M_{2}, \ldots$ be the cores of the handles $h_{1}, h_{2}, \ldots$, chosen such that their union is a smoothly embedded $C W$ complex inside $X$. Since we have not assumed that our handlebody is finite, we can not ask for the ordering of the handles with regards to their indices. However, due to local compactness we can, and will, ask that when a handle $h_{j}$ with core $M_{j}$ is being attached, all handles whose core discs intersect the boundary $\partial M_{j}$ have already been attached in earlier steps. We can also assume that $\partial M_{j}$ consists only of the core discs of handles of lower indices. We can now proceed with the induction as in the proof of Theorem6.1, but with the following modifications: 
(1) When a 2-handle is attached with a wrong framing, we insert the right number of kinks to its core disc (inside $X$ ) in order to change the framing coefficient to -1 , thereby insuring that we can extend $J$ to a Stein structure in a tubular strongly pseudoconvex neighborhood of the immersed disc. (The disc is totally real in this structure, with a special double point (4.1) at each kink.)

(2) Each time before proceeding to the next handle $h_{j+1}$, we perform one more step on each of the kinked discs appearing in the sequence before. More precisely, we add a new kinked disc which cancels the superfluous loop at the self-intersection point introduced in the previous step. (Of course this new kinked disc introduces a new superfluous loop which will have to be cancelled in the subsequent step.)

The first condition is essential since we need to build a manifold that is Stein. The second condition insures that each handle is properly worked upon, thereby producing a Casson tower at every place where a kink was made in the initial 2-disc. At every step we also apply Lemma 5.1 to approximate the given map, which has already been made holomorphic in a tubular strongly pseudoconvex neighborhood of our partial (finite) subcomplex, by a map holomorphic in a tubular neighborhood of the previous domain with all core discs that have been added at the given step.

The proof can now be concluded as in Theorem 6.1. We construct an increasing sequence of Stein domains $X_{1} \subset X_{2} \subset \cdots$ inside the original smooth 4-manifold $X$, each of them Runge in the next one, together with a sequence of maps $f_{j}: X \rightarrow Y(j=1,2, \ldots)$ such that $f_{j}$ is holomorphic on $X_{j}$, it approximates $f_{j-1}$ uniformly on $X_{j-1}$, and is homotopic to $f_{j-1}$ by a homotopy which is holomorphic and uniformly close to $f_{j-1}$ on $X_{j-1}$. The Runge property insures that the limit manifold $X^{\prime}=\cup_{j} X_{j}$ is Stein with respect to the limit complex structure and, by the construction, it is homeomorphic to $X$. (It is diffeomorphic to $X$ if no Casson handles were used in the construction.) A small ambient topological deformation moves the initial CW complex (made of cores of the attached handles) into $X^{\prime}$; see [28] for more details. By construction the limit map $f^{\prime}=\lim _{j \rightarrow \infty} f_{j}: X^{\prime} \rightarrow$ $Y$ is holomorphic, and the map $f^{\prime} \circ h: X \rightarrow Y$ is homotopic to $f$.

The same proof applies to any smoothly embedded 2-complex $M$ inside $X$ : After a small ambient topological deformation we find a new embedding $M^{\prime} \hookrightarrow X$ with a Stein thickening $X^{\prime} \subset X$ such that a given continuous map $M \rightarrow Y$ admits a holomorphic representative $X^{\prime} \rightarrow Y$.

Remark 7.4. Whenever a handle is wrongly attached, the above process is never finite. The reason is that in the standard kink $K$, the disc $\Delta$ needed to be added to reconstruct the original manifold requires exactly one positive kink in order to be able to extend the Stein structure to its neighborhood. 
Remark 7.5. The complex structure in the above proof was built by stepwise extension across handles. If one begins with an almost complex structure $J$ defined on all of $X$, the Stein structure constructed by this process is in general not homotopic to $J$ (compare with Proposition 3.3). Below we shall give a different construction which will produce a Stein structure homotopic to the given initial complex structure on $X$. With a bit more work as in 27] one can also show that every relative cohomology class in $H^{2}(X, W ; \mathbb{Z})$ which reduces $\bmod 2$ to the second Stiefel-Whitney class $w_{2}(T X) \in H^{2}\left(X, W ; \mathbb{Z}_{2}\right)$ can be obtained as a reduction of a cohomology class of $c_{1}(X, J)$ for an appropriate $J$ in Theorem 7.1 .

Proof of Theorem 1.2 for $n=2$. We assume that $(X, J)$ is a complex surface with an integrable (not necessarily Stein) structure $J$, and with a correct handlebody structure. By a modification of the proof given above we shall construct an increasing sequence of domains $X_{1} \subset X_{2} \subset \ldots$ in $X$ and $J$-holomorphic maps $f_{j}: X_{j} \rightarrow Y$ such that each $X_{j}$ is a $J$-Stein domain in $X$ and the other properties are as before. The proof which we shall give is similar to the construction of Gompf [31, the difference being that we do everything by using the special kink $K$ introduced above.

We need to recall a few basic facts about the complex points of real surfaces in complex surfaces. Let $M$ be a closed real surface smoothly immersed in a complex surface $X$. A generic such $M$ has finitely many complex points $p \in M$ (i.e., points where the tangent space $T_{p} M$ is a complex line in $T_{p} X$ ) and finitely many transverse double points; in addition, no complex point is a double point of $M$. Locally near a complex point $p$ the surface $M$ is given in suitable local holomorphic coordinates $(z, w)$, with $z(p)=w(p)=0$, by an equation $w=|z|^{2}+\lambda\left(z^{2}+\bar{z}^{2}\right)+o\left(|z|^{2}\right)$ for a unique $\lambda \geq 0$ (Bishop [3]). The point $p$ is elliptic if $\lambda<1 / 2$ and hyperbolic if $\lambda>1 / 2$; the degenerate case $\lambda=1 / 2$ does not arise for a generic $M$. At an elliptic point $M$ has a nontrivial local envelope of holomorphy consisting of a family of small analytic discs (the so called Bishop discs, [3]). At a hyperbolic point $M$ is locally holomorphically convex 24] and it admits a basis of tubular Stein neighborhoods [53].

If $M$ is oriented, one further divides its complex points into positive and negative ones, depending on whether the standard complex line orientation of $T_{p} M$ agrees or disagrees with the chosen orientation of $M$. Let $e_{ \pm}(M)$ resp. $h_{ \pm}(M)$ indicate the numbers of elliptic resp. hyperbolic points in each orientation class. The Lai indices $I_{ \pm}(M)=e_{ \pm}(M)-h_{ \pm}(M)$ are invariant under a regular homotopy of the immersion $M \rightarrow X$ and satisfy

$$
2 I_{ \pm}(M)=\chi(M) \pm\left\langle c_{1}(X),[M]\right\rangle+[M]^{2}-2 d(M) .
$$

Here $\chi(M)$ is the Euler number of $M,[M] \in H_{2}(X ; \mathbb{Z})$ is the homology class of $M, c_{1}(X)=c_{1}(T X)$ is the first Chern class of the tangent bundle $T X$, and $d(M)$ is the algebraic number of self-intersection points of $M$ counted with oriented intersection indices (see [3], 4], [10, [16], 43], 47], [56]). 
A basic fact proved by Eliashberg and Harlamov [10] (see also [15] and [47]) is that one can cancel a pair of an elliptic and a hyperbolic point in the same orientation class by a $\mathcal{C}^{0}$-small isotopy supported in a neighborhood of a suitably chosen embedded arc in $M$ connecting these two points. If $I_{ \pm}(M) \leq 0$, we can cancel all elliptic points and deform $M$ to an immersed surface with only special double points (4.1) which is either totally real (if $I_{ \pm}(M)=0$ ) or has only special hyperbolic points, given in local holomorphic coordinates by $w=z^{2}+\bar{z}^{2}$ [16, Theorem 1.2, p. 82]. By [16, Theorem $2.2]$ this new $M$ admits a basis of smoothly bounded Stein neighborhoods diffeomorphic to $M \times \mathbb{R}^{2}$, given as sublevel sets of a smooth plurisubharmonic function $\tau \geq 0$ which vanishes precisely on $M$ and has no critical points in a deleted neighborhood of $M$. (Locally at a special hyperbolic point $w=z^{2}+\bar{z}^{2}$ we can take $\tau(z, w)=\left|w-z^{2}-\bar{z}\right|^{2}$. It was shown in [53] how to find strongly pseudoconvex tubular neighborhoods of any surface with only hyperbolic complex points.)

By adding a positive kink to $M$ we do not change its homology class $[M]$ and its Euler number $\chi(M)$, but we increase by one the number of double points $d(M)$. Hence we see from (7.3) that a positive kink reduces each of the numbers $I_{ \pm}(M)$ by 1 . This can also be seen directly: If in local coordinates $(z, w)=(x+i y, u+i v)$ the double point is given by $R^{2} \cup i \mathbb{R}^{2}=\{(x+i u)(y+i v)=0\}$ (with the orientation reversed on the second summand), the perturbation $(x+i u)(y+i v)=2 \epsilon^{2}$ for $\epsilon>0$ is a smooth surface with two hyperbolic complex points $p_{1}=\epsilon(1+i,-1+i)$, $p_{2}=-p_{1}$. Adding a sufficient number of positive kinks and performing a $\mathcal{C}^{0}$. small regular homotopy as above we find an immersed surface $M$ with a basis of strongly pseudoconvex tubular Stein neighborhoods. For this argument we do not need the formula (7.3), it suffices to know that a positive kink reduces each of the numbers $I_{ \pm}$by one so that eventually they become nonpositive.

The same argument holds for a compact surface $M \subset X \backslash W$ with boundary attached to $\Sigma=\partial W$ along a Legendrian curve - after kinking it enough times we can find an isotopy, fixed near $\partial M$, to a new surface with only special hyperbolic points and special double points. (If $M$ is a disc, it can be seen that $I_{+}(M)+I_{-}(M)=[\beta]-T B+1$, but this will not be used.)

We also note that a continuous function on $M$ can be uniformly approximated by functions holomorphic in a neighborhood of any hyperbolic complex point $p \in M$. If $M$ only has hyperbolic complex points then we can approximate each continuous function on $M$ uniformly by holomorphic functions in a Stein neighborhood of $M$. It is now easily seen that Theorem 4.1 still holds (with uniform approximation on $M$ ) in the presence of hyperbolic points. The existence of Stein neighborhoods of the graph of $f$ in $X \times Y$, needed in the proof of Theorem 4.1, is easily insured by chosing $f$ to be constant in a small neighborhood of each hyperbolic point. 
We can now complete the proof of Theorem 1.2 when $\operatorname{dim}_{\mathbb{R}} X=4$. Assume that $X$ is obtained from a strongly $J$-pseudoconvex domain $\bar{W} \subset X$ by successively attaching handles $h_{1}, h_{2}, \ldots$ with core discs $M_{1}, M_{2}, \ldots$, where the ordering of these handles satisfies the same condition as in the proof of Theorem 7.1. (We may begin with $W=\emptyset$.) We shall use the same notation as in the proof of Theorem 1.2 for $\operatorname{dim}_{\mathbb{R}} X \neq 4$ (see $\S 6$ ), beginning with $W_{0}=W$ and $f_{0}=f$. The complex structure on $X$ will not change during the proof.

In the inductive step we have a smoothly bounded, strongly pseudoconvex domain $W_{j} \subset X$ and a map $f_{j}: X \rightarrow Y$ which is $J$-holomorphic in a neighborhod of $\bar{W}_{j}$. (The set $W_{j}$ is a tubular neighborhood of the union of $\bar{W}$ with the cores of handles attached in the earlier steps; since these cores may have received kinks, $W_{j}$ does not have the correct homeomorphic type, but this will be corrected in the limit by the Casson handles resulting from the construction.) We now attach to $\bar{W}_{j}$ the next handle in the sequence. As before, attaching a 1-handle does not pose a problem. For a 2-handle $h$ we first make sure that the boundary of its core $M$ is a Legendrian curve in $\partial W_{j}$, and then we add enough positive kinks to algebraically cancel off all elliptic points on the core disc $M$ as explained above. We denote this new immersed disc by $M^{\prime}$. After a $\mathcal{C}^{0}$-small regular homotopy fixing the boundary $\partial M^{\prime}$ we can assume that $\bar{W}_{j} \cup M^{\prime}$ has a basis of tubular, strongly pseudoconvex Stein neighborhoods in $X$. We also add to $\bar{W}_{j}$ a new trivializing kinky disc at each of the kinks from the earlier stages of the construction, making sure that the conditions (1) and (2) in the proof of Theorem 7.1 are satisfied. These additional kinky discs $\Delta_{1}, \ldots, \Delta_{k}$ can be chosen such that $L_{j}:=\bar{W}_{j} \cup M^{\prime} \cup\left(\cup_{l=1}^{k} \Delta_{l}\right)$ admits a basis of tubular, strongly pseudoconvex, Stein neighborhoods in $X$. Using Lemma 5.1 we approximate $f_{j}$ uniformly on $L_{j}$ by a map $f_{j+1}: X \rightarrow Y$ which is holomorphic in a neighborhood of $L_{j}$. Choosing a small strongly pseudoconvex tubular neighborhood $W_{j+1} \supset L_{j}$ contained in the domain of holomorphicity of $f_{j+1}$ completes the induction step. In the limit we obtain a holomorphic map $f^{\prime}: \Omega \rightarrow Y$ on the Stein domain $\Omega=\cup_{j} W_{j} \subset X$ with the stated properties.

Corollary 7.3 is proved by applying the construction, described in the proof of Theorem 1.2, to an embedded CW 2-complex in $X$.

Remark 7.6. By a small addition to the above argument it is possible to change every real surface $M$, attached from the outside to a strongly pseudoconvex domain $W \subset X$ along a Legendrian link, to a totally real immersed surface all of whose double points are positive kinks. Indeed, by an isotopy of $\partial M \hookrightarrow \partial W$ to another Legendrian link one can insure that $I_{+}(M)-I_{-}(M)=0$. (This difference equals the rotation number of the canonical contact extension of the Legendrian link $\partial M \subset \partial W$ which can be made equal to any given number by isotopies; see [7] and [27].) If $I_{+}(M)=$ $I_{-}(M)>0$, we add this many positive kinks and reduce $I_{ \pm}(M)$ to 0 , so our disc becomes totally real after a small isotopy. If $I_{+}(M)=I_{-}(M)<0$, we 
can increase them to zero by adding left spirals to $\partial M$ [27], again making $M$ totally real. In this way one can avoid having to deal with hyperbolic complex points at the cost of using Legendrian knot theory.

Acknowledgements. We thank Yakov Eliashberg whose help was essential in proving Lemma 3.1 for handles of maximal dimension, Robert Gompf for very helpful communication regarding his works [27, 28] on Stein surfaces, Petar Pavešić and Sašo Strle for their help with Proposition 3.3, and Irena Majcen for introducing us to drawing pictures with pstricks.

\section{REFERENCES}

1. Andreotti, A., Fraenkel, T.: The Lefshetz theorem on hyperplane sections. Ann. Math. 69 (2), 713-717 (1959)

2. Arnol'd, V.: Lagrangian and Legendrian cobordisms. Funct. Anal. Appl. 14, 167-177 (1980)

3. Bishop, E.: Differentiable manifolds in complex Euclidean spaces. Duke Math. J. 32, 1-21 (1965)

4. Chern, S. S., Spanier, E.: A theorem on orientable surfaces in four-dimensional space. Comm. Math. Helv. 25, 205-209 (1951)

5. Costantino, F.: Stein domains and branched shadows of 4-manifolds. Preprint (2005)

6. Duchamp, T.: The classification of Legendre immersions. Preprint (1984)

7. Eliashberg, Y.: Topological characterization of Stein manifolds of dimension $>2$. Internat. J. Math. 1, 29-46 (1990)

8. Eliashberg, Y.: Classification of overtwisted contact structures on 3-manifolds. Invent. Math. 98, 623-637 (1989)

9. Eliashberg, Y., Gromov, M.: Nonsingular maps of Stein manifolds. Func. Anal. Appl. 5, 82-83 (1971)

10. Eliashberg, Y., Harlamov, V.: Some remarks on the number of complex points of a real surface in a complex one. In: Proc. LITC-82, 143-148 (1982)

11. Eliashberg, Y., Mishachev, N., Introduction to the $h$-principle. Graduate Studies in Math. 48, Amer. Math. Soc., Providence (2002)

12. Fornæss, J. E., Stout, E. L.: Spreading polydiscs on complex manifolds. Amer. J. Math. 99, 933-960 (1977)

13. Forster, O., Ramspott, K. J.: Analytische Modulgarben und Endromisbündel. Invent. Math. 2, 145-170 (1966)

14. Forstnerič, F.: On totally real embeddings into $\mathbb{C}^{n}$. Expo. Math. 4, 243-255 (1986)

15. Forstnerič, F.: Complex tangents of real surfaces in complex surfaces. Duke Math. J. 67, 353-376 (1992)

16. Forstnerič, F.: Stein domains in complex surfaces, J. Geom. Anal. 13, 77-94 (2003)

17. Forstnerič, F.: Noncritical holomorphic functions on Stein manifolds. Acta Math. 191, 143-189 (2003)

18. Forstnerič, F.: Holomorphic submersions from Stein manifolds. Ann. Inst. Fourier 54, 1913-1942 (2004)

19. Forstnerič, F.: Runge approximation on convex sets implies Oka's property. Ann. Math. 163 (2), 689-707 (2006)

20. Forstnerič, F.: Extending holomorphic mappings from subvarieties in Stein manifolds. Ann. Inst. Fourier 55, 733-751 (2005)

21. Forstnerič, F.: Holomorphic flexibility properties of complex manifolds. Amer. J. Math. 128, 239-270 (2006)

22. Forstnerič, F., Kozak, J.: Strongly pseudoconvex handlebodies. J. Korean Math. Soc. 40, 727-745 (2003) 
23. Forstnerič, F., Prezelj, J.: Oka's principle for holomorphic fiber bundles with sprays. Math. Ann. 317, 117-154 (2000)

24. Forstnerič, F., Stout, E. L.: A new class of polynomially convex sets in $\mathbb{C}^{2}$. Ark. Mat. 29, 51-62 (1991)

25. Freedman, M.: The topology of four-dimensional manifolds. J. Diff. Geom. 17, 357$453(1982)$

26. Freedman, M. H., Quinn, F.: Topology of 4-manifolds. Princeton Mathematical Series 39, Princeton Univ. Press, Princeton (1990)

27. Gompf, R. E.: Handlebody construction of Stein surfaces. Ann. Math. 148 (2), 619 693 (1998)

28. Gompf, R. E.: Stein surfaces as open subsets of $\mathbb{C}^{2}$. J. Symplectic Geom. 3, 565-587 (2005)

29. Gompf, R. E., Stipsicz, A. I., 4-manifolds and Kirby calculus. Graduate Studies in Math. 20, Amer. Math. Soc., Providence (1999)

30. Grauert, H.: Approximationssätze für holomorphe Funktionen mit Werten in komplexen Räumen. Math. Ann. 133, 139-159 (1957)

31. Grauert, H.: Holomorphe Funktionen mit Werten in komplexen Lieschen Gruppen. Math. Ann. 133, 450-472 (1957)

32. Grauert, H.: Analytische Faserungen über holomorph-vollständigen Räumen. Math. Ann. 135, 263-273 (1958)

33. Grauert, H.: On Levi's problem. Ann. Math. 68 (2), 460-472 (1958)

34. Gromov, M.: Stable maps of foliations into manifolds. Izv. Akad. Nauk SSSR Ser. Mat. 33, 707-734 (1969) (Russian)

35. Convex integration of differential relations, I. Izv. Akad. Nauk SSSR Ser. Mat. 37, 329-343 (1973) (Russian). English transl.: Math. USSR Izv. 37 (1973)

36. Gromov, M.: Partial differential relations. Ergebnisse der Mathematik und ihrer Grenzgebiete (3) 9. Springer, Berlin New York (1986)

37. Gromov, M.: Oka's principle for holomorphic sections of elliptic bundles. J. Amer. Math. Soc. 2, 851-897 (1989)

38. Gunning, R. C., Narasimhan, R., Immersion of open Riemann surfaces. Math. Ann. 174, 103-108 (1967)

39. Hirsch, M., Immersions of manifolds. Trans. Amer. Math. Soc. 93, 242-276 (1959)

40. Hörmander, L.: An Introduction to Complex Analysis in Several Variables. Third ed. North Holland, Amsterdam (1990)

41. Hörmander, L., Wermer, J., Uniform approximations on compact sets in $\mathbb{C}^{n}$. Math. Scand. 23, 5-21 (1968)

42. Kronheimer, P. B., Mrowka, T. S.: Monopoles and contact structures. Invent. Math. 130, 209-255 (1997)

43. Lai, H. F.: Characteristic classes of real manifolds immersed in complex manifolds. Trans. Amer. Math. Soc. 172, 1-33 (1972)

44. Lefshetz, S.: L'analysis Situs et la Géometrie Algébrique. Paris (1924)

45. Lisca, P., Matić, G.: Tight contact structures and Seiberg-Witten invariants. Invent. Math. 129, 509-525 (1997)

46. Milnor, J.: Morse Theory. Ann. Math. Studies 51, Princeton Univ. Press, Princeton (1963)

47. Nemirovski, S.: Complex analysis and differential topology on complex surfaces. In: Uspekhi Mat. Nauk 54 (4), 47-74 (1999). English transl. in: Russian Math. Surveys 54 (4), 729-752 (1999)

48. Newlander, A., Nirenberg, L.: Complex analytic coordinates in almost complex manifolds. Ann. Math. 65 (2), 391-404 (1957)

49. Oka, K.: Sur les fonctions des plusieurs variables. III: Deuxième problème de Cousin. J. Sc. Hiroshima Univ. 9, 7-19 (1939) 
50. Ozsváth, P., Szabo, Z.: The symplectic Thom conjecture. Ann. Math. 151 (2), 93-124 (2000)

51. Phillips, A.: Submersions of open manifolds. Topology 6, 170-206 (1967)

52. Rosay, J.-P.: A counterexample related to Hartog's phenomenon (a question by E. Chirka). Michigan Math. J. 45, 529-535 (1998)

53. Slapar, M.: On Stein neighborhood basis of real surfaces. Math. Z. 247, 863-879 (2004)

54. Smale, S., The classification of immersions of spheres in Euclidean spaces. Ann. Math. 69 (2), 327-344 (1959)

55. Stout, E. L.: The Theory of Uniform Algebras. Bogden\&Quigley, Inc., Tarrytown-onHudson, New York (1971)

56. Webster, S.: Minimal surfaces in a Kähler surface. J. Diff. Geom. 20, 463-470 (1984)

57. Weinstein, A.: Lectures on Symplectic Manifolds. Reg. Conf. Ser. Math. 29, Amer. Math. Soc., Providence (1977)

58. Weinstein, A.: Contact surgery and symplectic handlebodies. Hokkaido Math. J. 20, 241-251 (1991)

59. Whitehead, G. W.: Elements of Homotopy Theory. Graduate Texts in Math. 61, Springer-Verlag, Berlin (1978)

60. Whitney, H.: The self-intersections of a smooth manifold in a $2 n$-space. Ann. Math. 45 (2), 220-246 (1944)

Institute of Mathematics, Physics and Mechanics, University of Luubluana, Jadranska 19, 1000 Luubluana, Slovenia

E-mail address: franc.forstneric@fmf.uni-lj.si, marko.slapar@fmf.uni-lj.si 